\title{
Supporting Information: On the Role of the Special Pair in Photosystems as a Charge Transfer Rectifier
}

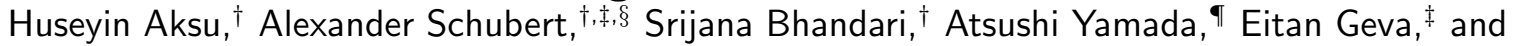
Barry D. Dunietz ${ }^{*, \dagger}$

Department of Chemistry and Biochemistry, Kent State University, Kent, OH 44242, USA, Department of Chemistry, University of Michigan, Ann Arbor, MI 48109, USA, and Center for Computational Sciences, University of Tsukuba, 1-1-1 Tennodai, Tsukuba, Ibaraki 305-8577 Japan

Received February 8, 2020; E-mail: bdunietz@kent.edu

\section{S2-S5: SI Tables and Figures. \\ S4-S8: X-Ray atoms coord. (Å).}

\footnotetext{
${ }^{\dagger}$ Department of Chemistry and Biochemistry, Kent State University, Kent, OH 44242, USA

${ }^{\ddagger}$ Department of Chemistry, University of Michigan, Ann Arbor, MI 48109, USA

ฯ Center for Computational Sciences, University of Tsukuba, 1-1-1 Tennodai, Tsukuba, Ibaraki 305-8577 Japan

${ }^{\S}$ Present address: The Institute of Physical Chemistry, FriedrichSchiller-University, Jena, Germany
} 
SI Table S1. Excited states of the special pair calculated with SRSH-PCM scheme at various dielectric constants. Structures are obtained in corresponding constant.

\begin{tabular}{|c|c|c|c|c|c|c|c|c|}
\hline$\epsilon$ & state & $\begin{array}{l}\text { main } \\
\text { configuration }\end{array}$ & $\begin{array}{l}\text { excitation } \\
\text { energy }(\mathrm{eV})\end{array}$ & $\begin{array}{l}\text { oscillator } \\
\text { strength }\end{array}$ & net charge & & & \\
\hline & $\mathrm{S}_{1}$ & $\mathrm{H} \rightarrow \mathrm{L}(0.80)$ & 1.8709 & 0.79 & 0.02 & & & \\
\hline & & $\mathrm{H}-1 \rightarrow \mathrm{L}+1(0.37)$ & & & & & & \\
\hline & $\mathrm{S}_{2}$ & $\mathrm{H} \rightarrow \mathrm{L}+1(0.67)$ & 1.9225 & 0.02 & 0.02 & & & \\
\hline GAS & & & & & & & & \\
\hline & $\mathrm{CT}_{1}$ & $\mathrm{H}-1 \rightarrow \mathrm{L}(-0.56)$ & 2.3459 & 0.00 & -0.46 & & & \\
\hline & & $\mathrm{H} \rightarrow \mathrm{L}(0.53)$ & & & & & $\mathrm{CT}_{1}$ & $\mathrm{H} \rightarrow \mathrm{L}(0.87)$ \\
\hline & & $\mathrm{H} \rightarrow \mathrm{L}+1(0.58)$ & & & & & & $\mathrm{H} \rightarrow \mathrm{L}+1(0.41)$ \\
\hline & $\mathrm{CT}_{2}$ & $\mathrm{H}-1 \rightarrow \mathrm{L}(0.89)$ & 2.4091 & 0.00 & 0.65 & & $\mathrm{CT}_{2}$ & $\mathrm{H} \rightarrow \mathrm{L}(-0.41)$ \\
\hline & $S_{1}$ & $\begin{array}{l}\mathrm{H} \rightarrow \mathrm{L}(-0.22) \\
\mathrm{H} \rightarrow \mathrm{L}(0.92)\end{array}$ & & & & & & $\mathrm{H} \rightarrow \mathrm{L}+1(0.80)$ \\
\hline & $\begin{array}{l}\mathrm{S}_{1} \\
\mathrm{~S}_{2}\end{array}$ & $\begin{array}{l}\mathrm{H} \rightarrow \mathrm{L}(0.92) \\
\mathrm{H} \rightarrow \mathrm{L}+1(0.92)\end{array}$ & $\begin{array}{l}1.6690 \\
1.7273\end{array}$ & 0.89 & -0.14 & 23 & & $\mathrm{H}-1 \rightarrow \mathrm{L}(-0.36)$ \\
\hline 1.2 & & & & 0.05 & 0.01 & 2.3 & $S_{1}$ & $\mathrm{H}-1 \rightarrow \mathrm{L}+1(0.94)$ \\
\hline & $\mathrm{CT}_{1}$ & $\mathrm{H}-1 \rightarrow \mathrm{L}(0.88)$ & 1.9992 & 0.10 & -0.69 & & & $\mathrm{H} \rightarrow \mathrm{L}(0.26)$ \\
\hline & & $\mathrm{H}-1 \rightarrow \mathrm{L}+1(0.27)$ & & & & & $\mathrm{S}_{2}$ & $\mathrm{H} \rightarrow \mathrm{L}+1(0.40)$ \\
\hline & $\mathrm{CT}_{2}$ & $\mathrm{H}-1 \rightarrow \mathrm{L}(-0.27)$ & 2.0858 & 0.08 & 0.78 & & & $\mathrm{H}-1 \rightarrow \mathrm{L}(0.90)$ \\
\hline & & $\mathrm{H}-1 \rightarrow \mathrm{L}+1(0.94)$ & & & & & $\mathrm{CT}_{1}$ & $\mathrm{H} \rightarrow \mathrm{L}(0.85)$ \\
\hline & $\mathrm{S}_{1}$ & $\mathrm{H} \rightarrow \mathrm{L}(0.94)$ & 1.6603 & 0.81 & -0.20 & & & $\mathrm{H} \rightarrow \mathrm{L}+1(0.42)$ \\
\hline & $\mathrm{S}_{2}$ & $\mathrm{H} \rightarrow \mathrm{L}+1(0.95)$ & 1.7193 & 0.07 & 0.02 & & $\mathrm{CT}_{2}$ & $\mathrm{H} \rightarrow \mathrm{L}(-0.43)$ \\
\hline 1.3 & & $\mathrm{H}-1 \rightarrow \mathrm{L}(0.91)$ & & & & & & $\mathrm{H} \rightarrow \mathrm{L}+1(0.78)$ \\
\hline & $U 1_{1}$ & $\begin{array}{l}\mathrm{H}-1 \rightarrow \mathrm{L}+1(0.27)\end{array}$ & 1.9606 & 0.13 & -0.62 & 2.4 & & $\mathrm{H}-\mathrm{I} \rightarrow \mathrm{L}(-0.38)$ \\
\hline & $\mathrm{CT}_{2}$ & $\begin{array}{l}\mathrm{H}-1 \rightarrow \mathrm{L}(-0.27) \\
\mathrm{H}-1 \rightarrow \mathrm{L}+1(0.91)\end{array}$ & 2.0395 & 0.10 & 0.75 & & $\mathrm{~S}_{1}$ & $\begin{array}{l}\mathrm{H}-1 \rightarrow \mathrm{L}+1(0.93) \\
\mathrm{H} \rightarrow \mathrm{L}(0.28)\end{array}$ \\
\hline & $\mathrm{S}_{1}$ & $\mathrm{H} \rightarrow \mathrm{L}(0.96)$ & 1.6496 & 0.72 & -0.27 & & $\mathrm{~S}_{2}$ & $\mathrm{H} \rightarrow \mathrm{L}+1(0.41)$ \\
\hline & $\mathrm{S}_{2}$ & $\mathrm{H} \rightarrow \mathrm{L}+1(0.97)$ & 1.7101 & 0.11 & 0.09 & & & $\mathrm{H}-1 \rightarrow \mathrm{L}(0.89)$ \\
\hline 1.4 & & & & & & & $\mathrm{CT}_{1}$ & $\mathrm{H} \rightarrow \mathrm{L}(0.82)$ \\
\hline & $\mathrm{CT}_{1}$ & $\mathrm{H}-1 \rightarrow \mathrm{L}(0.93)$ & 1.9327 & 0.15 & -0.55 & & & $\mathrm{H} \rightarrow \mathrm{L}+1(0.46)$ \\
\hline & & $\mathrm{H}-1 \rightarrow \mathrm{L}+1(0.27)$ & & & & & $\mathrm{CT}_{2}$ & $\mathrm{H} \rightarrow \mathrm{L}(-0.45)$ \\
\hline & $\mathrm{CT}_{2}$ & $\mathrm{H}-1 \rightarrow \mathrm{L}(-0.27)$ & 2.0025 & 0.13 & 0.72 & & & $\mathrm{H} \rightarrow \mathrm{L}+1(0.74)$ \\
\hline & & $\mathrm{H}-1 \rightarrow \mathrm{L}+1(0.94)$ & & & & & & $\mathrm{H}-1 \rightarrow \mathrm{L}(-0.39)$ \\
\hline & $\mathrm{S}_{1}$ & $\mathrm{H} \rightarrow \mathrm{L}(0.98)$ & 1.6384 & 0.64 & -0.35 & 2.6 & & \\
\hline 1.5 & $\mathrm{~S}_{2}$ & $\mathrm{H} \rightarrow \mathrm{L}+1(0.98)$ & 1.7001 & 0.14 & 0.14 & & $\mathrm{~S}_{1}$ & $\begin{array}{l}\mathrm{H}-1 \rightarrow \mathrm{L}+1(0.91) \\
\mathrm{H} \rightarrow \mathrm{L}(0.33)\end{array}$ \\
\hline & $\mathrm{CT}_{1}$ & $\begin{array}{l}\mathrm{H}-1 \rightarrow \mathrm{L}(0.94) \\
\mathrm{H}-1 \rightarrow \mathrm{L}+1(0.27)\end{array}$ & 1.9086 & 0.18 & -0.48 & & $\mathrm{~S}_{2}$ & $\begin{array}{l}\mathrm{H} \rightarrow \mathrm{L}+1(0.43) \\
\mathrm{H}-1 \rightarrow \mathrm{L}(0.87)\end{array}$ \\
\hline & $\mathrm{CT}_{2}$ & $\begin{array}{l}\mathrm{H}-1 \rightarrow \mathrm{L}(-0.27) \\
\mathrm{H}-1 \rightarrow \mathrm{L}+1(0.94)\end{array}$ & 1.9714 & 0.16 & 0.67 & & $\mathrm{CT}_{1}$ & $\begin{array}{l}\mathrm{H} \rightarrow \mathrm{L}(0.80) \\
\mathrm{H} \rightarrow \mathrm{L}+1(0.48)\end{array}$ \\
\hline & $\mathrm{S}_{1}$ & $\mathrm{H} \rightarrow \mathrm{L}(0.98)$ & 1.6261 & 0.56 & -0.42 & & $\mathrm{CT}_{2}$ & $\mathrm{H} \rightarrow \mathrm{L}(-0.47)$ \\
\hline & $\mathrm{S}_{2}$ & $\mathrm{H} \rightarrow \mathrm{L}+1(0.98)$ & 1.6904 & 0.16 & 0.20 & & & $\mathrm{H} \rightarrow \mathrm{L}+1(0.72)$ \\
\hline 1.6 & & & & & & & & $\mathrm{H}-1 \rightarrow \mathrm{L}(-0.40)$ \\
\hline & $\mathrm{CT}_{1}$ & $\mathrm{H}-1 \rightarrow \mathrm{L}(0.94)$ & 1.8911 & 0.20 & -0.42 & & & $\mathrm{H}-1 \rightarrow \mathrm{L}+1(0.31)$ \\
\hline & & $\mathrm{H}-1 \rightarrow \mathrm{L}+1(0.27)$ & & & & 2.8 & & \\
\hline & $\mathrm{CT}_{2}$ & $\begin{array}{l}\mathrm{H}-1 \rightarrow \mathrm{L}(-0.27) \\
\mathrm{H}-1 \rightarrow \mathrm{L}+1(0.94)\end{array}$ & 1.9458 & 0.20 & 0.62 & & $\mathrm{~S}_{1}$ & $\begin{array}{l}\mathrm{H}-1 \rightarrow \mathrm{L}+1(0.89) \\
\mathrm{H} \rightarrow \mathrm{L}(0.36)\end{array}$ \\
\hline & $\mathrm{S}_{1}$ & $\mathrm{H} \rightarrow \mathrm{L}(0.98)$ & 1.6128 & 0.49 & -0.48 & & $\mathrm{~S}_{2}$ & $\mathrm{H} \rightarrow \mathrm{L}+1(0.44)$ \\
\hline & $\mathrm{S}_{2}$ & $\mathrm{H} \rightarrow \mathrm{L}+1(0.98)$ & 1.6840 & 0.18 & 0.27 & & & $\mathrm{H}-1 \rightarrow \mathrm{L}(0.86)$ \\
\hline 1.7 & & & & & & & $\mathrm{CT}_{1}$ & $\mathrm{H} \rightarrow \mathrm{L}(0.78)$ \\
\hline & $\mathrm{CT}_{1}$ & $\mathrm{H}-1 \rightarrow \mathrm{L}(0.94)$ & 1.8769 & 0.22 & -0.37 & & & $\mathrm{H} \rightarrow \mathrm{L}+1(0.50)$ \\
\hline & & $\mathrm{H}-1 \rightarrow \mathrm{L}+1(0.28)$ & & & & & $\mathrm{CT}_{2}$ & $\mathrm{H} \rightarrow \mathrm{L}(-0.48)$ \\
\hline & $\mathrm{CT}_{2}$ & $\mathrm{H}-1 \rightarrow \mathrm{L}(-0.28)$ & 1.9240 & 0.22 & 0.57 & & & $\mathrm{H} \rightarrow \mathrm{L}+1(0.69)$ \\
\hline & $\mathrm{S}_{1}$ & $\mathrm{H} \rightarrow \mathrm{L}(0.50)$ & 1.6035 & 0.47 & -0.54 & & & $\mathrm{H}-1 \rightarrow \mathrm{L}(-0.41)$ \\
\hline & & $\mathrm{H} \rightarrow \mathrm{L}+1(0.77)$ & & & & & & $\mathrm{H}-1 \rightarrow \mathrm{L}+1(0.34)$ \\
\hline & & $\mathrm{H}-1 \rightarrow \mathrm{L}+1(0.32)$ & & & & 3.0 & & \\
\hline & $\mathrm{S}_{2}$ & $\mathrm{H} \rightarrow \mathrm{L}(0.83)$ & 1.6737 & 0.15 & 0.38 & & $\mathrm{~S}_{1}$ & $\mathrm{H} \rightarrow \mathrm{L}(0.39)$ \\
\hline 1.8 & & $\mathrm{H} \rightarrow \mathrm{L}+1(-0.48)$ & & & & & & $\mathrm{H}-1 \rightarrow \mathrm{L}+1(0.88)$ \\
\hline 1.0 & $\mathrm{CT}_{1}$ & $\mathrm{H} \rightarrow \mathrm{L}+1(-0.40)$ & 1.8531 & 0.24 & -0.35 & & $\mathrm{~S}_{2}$ & $\begin{array}{l}\mathrm{H}-1 \rightarrow \mathrm{L}+1(0.85) \\
\mathrm{H} \rightarrow \mathrm{L}+1(0.46)\end{array}$ \\
\hline & & $\mathrm{H}-1 \rightarrow \mathrm{L}+1(0.86)$ & & & & & $\mathrm{CT}_{1}$ & $\mathrm{H} \rightarrow \mathrm{L}(0.74)$ \\
\hline & & $\mathrm{H} \rightarrow \mathrm{L}+1(0.40)$ & & & & & & $\mathrm{H} \rightarrow \mathrm{L}+1(0.52)$ \\
\hline & $\mathrm{CT}_{2}$ & $\mathrm{H}-1 \rightarrow \mathrm{L}(0.91)$ & 1.8716 & 0.25 & 0.42 & & & $\mathrm{H}-1 \rightarrow \mathrm{L}(0.36)$ \\
\hline & & $\mathrm{H}-1 \rightarrow \mathrm{L}+1(-0.31)$ & & & & & $\mathrm{CT}_{2}$ & $\mathrm{H}-1 \rightarrow \mathrm{L}(-0.38)$ \\
\hline & $\mathrm{S}_{1}$ & $\mathrm{H} \rightarrow \mathrm{L}(0.42)$ & 1.5986 & 0.42 & -0.58 & & & $\mathrm{H} \rightarrow \mathrm{L}+\mathrm{i}(0.64)$ \\
\hline & & $\mathrm{H} \rightarrow \mathrm{L}+1(0.77)$ & & & & & & $\mathrm{H} \rightarrow \mathrm{L}(0.48)$ \\
\hline & & $\mathrm{H}-1 \rightarrow \mathrm{L}+1(0.36)$ & & & & $4.0 \ldots$ & & \\
\hline & $\mathrm{S}_{2}$ & $\mathrm{H} \rightarrow \mathrm{L}(0.85)$ & 1.6611 & 0.14 & 0.45 & & $\mathrm{~S}_{1}$ & $\mathrm{H} \rightarrow \mathrm{L}(0.46)$ \\
\hline & & $\mathrm{H} \rightarrow \mathrm{L}+1(-0.45)$ & & & & & & $\mathrm{H}-1 \rightarrow \mathrm{L}+1(0.84)$ \\
\hline 1.9 & & & & & & & $\mathrm{~S}_{2}$ & $\mathrm{H}-1 \rightarrow \mathrm{L}(0.84)$ \\
\hline & $\mathrm{CT}_{1}$ & $\mathrm{H} \rightarrow \mathrm{L}+1(-0.43)$ & 1.8308 & 0.29 & -0.31 & & & $\mathrm{H} \rightarrow \mathrm{L}+1(0.49)$ \\
\hline & & $\mathrm{H}-1 \rightarrow \mathrm{L}+1(0.86)$ & & & & & $\mathrm{CT}_{1}$ & $\mathrm{H} \rightarrow \mathrm{L}(0.61)$ \\
\hline & $\mathrm{CT}_{2}$ & $\mathrm{H}-1 \rightarrow \mathrm{L}(0.94)$ & 1.8579 & 0.25 & 0.36 & & & $\mathrm{H} \rightarrow \mathrm{L}+1(0.61)$ \\
\hline & & $\mathrm{H}-1 \rightarrow \mathrm{L}+1(-0.23)$ & & & & & & $\mathrm{H}-1 \rightarrow \mathrm{L}(0.42)$ \\
\hline & $\mathrm{S}_{1}$ & $\mathrm{H} \rightarrow \mathrm{L}(0.48)$ & 1.5911 & 0.39 & -0.60 & & $\mathrm{CT}_{2}$ & $\mathrm{H}-1 \rightarrow \mathrm{L}(0.40)$ \\
\hline & & $\mathrm{H} \rightarrow \mathrm{L}+1(0.75)$ & & & & & & $\mathrm{H} \rightarrow \mathrm{L}+\mathrm{1}(0.52)$ \\
\hline & & $\mathrm{H}-1 \rightarrow \mathrm{L}+1(0.39)$ & & & & & & $\mathrm{H} \rightarrow \mathrm{L}(0.56)$ \\
\hline & $\mathrm{S}_{2}$ & $\mathrm{H} \rightarrow \mathrm{L}(0.86)$ & 1.6484 & 0.12 & 0.49 & $10.0 \ldots$ & & \\
\hline & & $\mathrm{H} \rightarrow \mathrm{L}+1(-0.44)$ & & & & & $\mathrm{S}_{1}$ & $\mathrm{H} \rightarrow \mathrm{L}(0.53)$ \\
\hline 2.0 & & & & & & & & $\mathrm{H}-1 \rightarrow \mathrm{L}+1(0.75)$ \\
\hline & $\mathrm{CT}_{1}$ & $\mathrm{H} \rightarrow \mathrm{L}+1(-0.46)$ & 1.8278 & 0.34 & -0.28 & & $\mathrm{~S}_{2}$ & $\mathrm{H}-1 \rightarrow \mathrm{L}(0.59)$ \\
\hline & & $\mathrm{H}-1 \rightarrow \mathrm{L}+1(0.86)$ & & & & & & $\mathrm{H} \rightarrow \mathrm{L}+\mathrm{1}(0.35)$ \\
\hline & $\mathrm{CT}_{2}$ & $\mathrm{H}-1 \rightarrow \mathrm{L}(0.96)$ & 1.8489 & 0.24 & 0.31 & & $\mathrm{CT}_{1}$ & $\mathrm{H} \rightarrow \mathrm{L}+1(0.69)$ \\
\hline & $\mathrm{CT}_{1}$ & $\mathrm{H} \rightarrow \mathrm{L}(0.90)$ & 1.5772 & 0.30 & -0.69 & & & $\mathrm{H} \rightarrow \mathrm{L}(0.48)$ \\
\hline & & $\mathrm{H} \rightarrow \mathrm{L}+1(0.37)$ & & & & & & $\mathrm{H}-1 \rightarrow \mathrm{L}(0.45)$ \\
\hline & $\mathrm{CT}_{2}$ & $\mathrm{H} \rightarrow \mathrm{L}(-0.38)$ & 1.6267 & 0.15 & 0.55 & & $\mathrm{CT}_{2}$ & $\mathrm{H} \rightarrow \mathrm{L}(0.64)$ \\
\hline & & $\mathrm{H} \rightarrow \mathrm{L}+1(0.84)$ & & & & & & $\mathrm{H} \rightarrow \mathrm{L}+1(0.40)$ \\
\hline & & $\mathrm{H}-1 \rightarrow \mathrm{L}(-0.33)$ & & & & & & $\mathrm{H}-1 \rightarrow \mathrm{L}+1(0.51)$ \\
\hline 2.1 & & & & & & & & $\mathrm{H}-1 \rightarrow \mathrm{L}(0.42)$ \\
\hline & $\mathrm{S}_{1}$ & $\mathrm{H}-1 \rightarrow \mathrm{L}+1(0.97)$ & 1.7789 & 0.62 & 0.13 & 78 & & \\
\hline & $\mathrm{S}_{2}$ & $\mathrm{H} \rightarrow \mathrm{L}+1(0.36)$ & 1.8071 & 0.02 & -0.01 & & $\mathrm{~S}_{1}$ & $\mathrm{H}-1 \rightarrow \mathrm{L}+1(0.74)$ \\
\hline & & $\mathrm{H}-1 \rightarrow \mathrm{L}(0.92)$ & & & & & & $\mathrm{H} \rightarrow \mathrm{L}(-0.56)$ \\
\hline & $\mathrm{CT}_{1}$ & $\mathrm{H} \rightarrow \mathrm{L}(0.88)$ & 1.5708 & 0.27 & -0.73 & & $\mathrm{~S}_{2}$ & $\mathrm{H}-1 \rightarrow \mathrm{L}(0.74)$ \\
\hline & & $\mathrm{H} \rightarrow \mathrm{L}+1(0.38)$ & & & & & & $\mathrm{H} \rightarrow \mathrm{L}+1(-0.55)$ \\
\hline & $\mathrm{CT}_{2}$ & $\mathrm{H} \rightarrow \mathrm{L}(-0.39)$ & 1.6185 & 0.15 & 0.61 & & & \\
\hline & & $\mathrm{H} \rightarrow \mathrm{L}+1(0.82)$ & & & & & & \\
\hline & & $\mathrm{H}-1 \rightarrow \mathrm{L}(-0.35)$ & & & & & & \\
\hline 2.2 & $\mathrm{~S}_{1}$ & & 1.7948 & 0.64 & 0.13 & & & \\
\hline & & $\mathrm{H} \rightarrow \mathrm{L}(0.23)$ & & & & & & \\
\hline & $\mathrm{S}_{2}$ & $\mathrm{H} \rightarrow \mathrm{L}+1(0.38)$ & 1.8069 & 0.03 & -0.03 & & & \\
\hline
\end{tabular}


SI Table S2. Excited states energies at $\epsilon=2.0$ for the dimers of Figure $5(B)$ and $5(C)$ where the distinctly oriented PFGs are removed. Figure 5(B) dimer exhibits same orientation as in Figure 5(A). In Figure 5(C), the monomers are shifted towards each other to obtain a stacked dimer geometry.

\begin{tabular}{ccccc}
\hline & state & $\begin{array}{c}\text { excitation } \\
\text { energy }(\mathrm{eV})\end{array}$ & $\begin{array}{c}\text { oscillator } \\
\text { strength }\end{array}$ & $\begin{array}{c}\text { net charge } \\
(e)\end{array}$ \\
\hline & $\mathrm{S}_{1}$ & 1.9371 & 0.91 & 0.00 \\
$5(\mathrm{~b})$ & $\mathrm{S}_{2}$ & 2.0131 & 0.00 & 0.00 \\
& & & & \\
& $\mathrm{CT}_{1}$ & 2.2277 & 0.00 & 0.61 \\
& $\mathrm{CT}_{2}$ & 2.2597 & 0.05 & -0.62 \\
\hline & $\mathrm{CT}_{1}$ & 2.0530 & 0.02 & -0.80 \\
& $\mathrm{CT}_{2}$ & 2.0675 & 0.02 & 0.76 \\
$5(\mathrm{c})$ & & & & \\
& $\mathrm{S}_{1}$ & 2.1392 & 0.00 & 0.05 \\
& $\mathrm{~S}_{2}$ & 2.2905 & 0.53 & 0.02 \\
\hline
\end{tabular}

SI Table S3. Diabatic states-based energy parameters and rates. Excited state $\left(\mathrm{S}_{D 1}\right)$, Electronic coupling ( $\left.V_{\mathrm{el}}\right)$, $\mathrm{CT}$ state energy, and $\mathrm{CT}$ optimized energies $\left(C T^{*}\right)$, driving force $(\Delta E)$, reorganization energy $\left(E_{r}^{\text {tot }}\right)$, activation energy $\left(E_{A}\right)$, Marcus $C T^{\prime}$ rate $\left(k_{\mathrm{M}}\right)$ and Fermi golden rule $\mathrm{CT}$ rate $\left(k_{\mathrm{FGR}}\right)$. For the $\mathrm{CT}$ states, $\mathrm{CT}_{A B}$ and $\mathrm{CT}_{B A}$ are provided at the first and second row, respectively, for each dielectric value.

\begin{tabular}{|c|c|c|c|c|c|c|c|c|c|}
\hline$\epsilon$ & $\begin{array}{l}\mathrm{S}_{D 1} \\
{[\mathrm{eV}]}\end{array}$ & $\begin{array}{c}\mathrm{CT} \\
{[\mathrm{eV}]}\end{array}$ & $\begin{array}{l}\mathrm{CT}^{*} \\
{[\mathrm{eV}]}\end{array}$ & $\begin{array}{c}V_{\mathrm{el}} \\
{[\mathrm{eV}]}\end{array}$ & $\begin{array}{r}|\Delta E| \\
{[\mathrm{eV}]}\end{array}$ & $\begin{array}{l}E_{r}^{\text {tot }} \\
{[\mathrm{eV}]}\end{array}$ & $\begin{array}{c}E_{\mathrm{a}} \\
{[\mathrm{eV}]}\end{array}$ & $\begin{array}{l}k_{\mathrm{M}} \\
{\left[s^{-1}\right]}\end{array}$ & $\begin{array}{l}k_{\mathrm{FGR}} \\
{\left[s^{-1}\right]}\end{array}$ \\
\hline \multirow[t]{2}{*}{1.2} & 1.7493 & 1.9136 & 1.6126 & 0.099 & 0.136 & 0.301 & 0.023 & $1.25 \times 10^{14}$ & $1.66 \times 10^{14}$ \\
\hline & & 2.0274 & 1.7264 & 0.102 & 0.022 & & 0.064 & $2.60 \times 10^{13}$ & $1.42 \times 10^{14}$ \\
\hline \multirow[t]{2}{*}{1.3} & 1.7506 & 1.8626 & 1.5536 & 0.097 & 0.188 & 0.309 & 0.012 & $1.79 \times 10^{14}$ & $1.81 \times 10^{14}$ \\
\hline & & 1.9739 & 1.6649 & 0.107 & 0.077 & & 0.044 & $6.40 \times 10^{13}$ & $1.52 \times 10^{14}$ \\
\hline \multirow[t]{2}{*}{1.4} & 1.7515 & 1.8185 & 1.5085 & 0.099 & 0.243 & 0.310 & 0.0036 & $2.56 \times 10^{14}$ & $1.80 \times 10^{14}$ \\
\hline & & 1.9289 & 1.6189 & 0.105 & 0.134 & & 0.0250 & $1.26 \times 10^{14}$ & $1.59 \times 10^{14}$ \\
\hline \multirow[t]{2}{*}{1.5} & 1.7531 & 1.7819 & 1.4709 & 0.098 & 0.282 & 0.311 & 0.0007 & $2.81 \times 10^{14}$ & $1.82 \times 10^{14}$ \\
\hline & & 1.8892 & 1.5782 & 0.102 & 0.175 & & 0.0147 & $1.78 \times 10^{14}$ & $1.49 \times 10^{14}$ \\
\hline \multirow[t]{2}{*}{1.6} & 1.7323 & 1.7257 & 1.4127 & 0.110 & 0.320 & 0.313 & 0.0001 & $3.62 \times 10^{14}$ & $1.84 \times 10^{14}$ \\
\hline & & 1.8215 & 1.5085 & 0.060 & 0.224 & & 0.0063 & $2.24 \times 10^{14}$ & $7.29 \times 10^{13}$ \\
\hline \multirow[t]{2}{*}{1.7} & 1.7370 & 1.7042 & 1.3912 & 0.104 & 0.341 & 0.313 & 0.0006 & $3.16 \times 10^{14}$ & $1.65 \times 10^{14}$ \\
\hline & & 1.7899 & 1.4769 & 0.063 & 0.260 & & 0.0022 & $1.19 \times 10^{14}$ & $6.62 \times 10^{13}$ \\
\hline \multirow[t]{2}{*}{1.8} & 1.7325 & 1.6902 & 1.3752 & 0.098 & 0.357 & 0.315 & 0.0014 & $2.71 \times 10^{14}$ & $1.45 \times 10^{14}$ \\
\hline & & 1.7624 & 1.4474 & 0.064 & 0.285 & & 0.0007 & $1.09 \times 10^{14}$ & $6.32 \times 10^{13}$ \\
\hline \multirow[t]{2}{*}{1.9} & 1.7454 & 1.6750 & 1.3600 & 0.095 & 0.385 & 0.315 & 0.0039 & $2.31 \times 10^{14}$ & $1.32 \times 10^{14}$ \\
\hline & & 1.7375 & 1.4225 & 0.064 & 0.323 & & 0.00001 & $1.22 \times 10^{14}$ & $6.27 \times 10^{13}$ \\
\hline \multirow[t]{2}{*}{2.0} & 1.7474 & 1.6614 & 1.3454 & 0.092 & 0.402 & 0.316 & 0.0059 & $2.01 \times 10^{14}$ & $1.20 \times 10^{14}$ \\
\hline & & 1.7160 & 1.4000 & 0.066 & 0.347 & & 0.0183 & $1.26 \times 10^{14}$ & $6.68 \times 10^{13}$ \\
\hline \multirow[t]{2}{*}{2.1} & 1.7326 & 1.6193 & 1.3023 & 0.058 & 0.430 & 0.317 & 0.0101 & $6.78 \times 10^{13}$ & $4.31 \times 10^{13}$ \\
\hline & & 1.6773 & 1.3603 & 0.074 & 0.373 & & 0.0025 & $1.48 \times 10^{14}$ & $8.14 \times 10^{13}$ \\
\hline \multirow[t]{2}{*}{2.2} & 1.7356 & 1.6086 & 1.2896 & 0.058 & 0.449 & 0.319 & 0.0132 & $5.98 \times 10^{13}$ & $3.91 \times 10^{13}$ \\
\hline & & 1.6637 & 1.3447 & 0.072 & 0.391 & & 0.0041 & $1.31 \times 10^{14}$ & $7.55 \times 10^{13}$ \\
\hline \multirow[t]{2}{*}{2.3} & 1.7386 & 1.5986 & 1.2786 & 0.056 & 0.460 & 0.320 & 0.0153 & $5.13 \times 10^{13}$ & $3.45 \times 10^{13}$ \\
\hline & & 1.6523 & 1.3323 & 0.070 & 0.406 & & 0.0058 & $1.16 \times 10^{14}$ & $6.92 \times 10^{13}$ \\
\hline \multirow[t]{2}{*}{2.4} & 1.7403 & 1.5897 & 1.2687 & 0.055 & 0.472 & 0.321 & 0.0196 & $4.18 \times 10^{13}$ & $3.18 \times 10^{13}$ \\
\hline & & 1.6414 & 1.3204 & 0.069 & 0.420 & & 0.0076 & $1.05 \times 10^{14}$ & $6.42 \times 10^{13}$ \\
\hline \multirow[t]{2}{*}{2.5} & 1.7422 & 1.5819 & 1.2619 & 0.053 & 0.480 & 0.320 & 0.0200 & $3.84 \times 10^{13}$ & $2.85 \times 10^{13}$ \\
\hline & & 1.6312 & 1.3112 & 0.068 & 0.431 & & 0.0002 & $9.43 \times 10^{13}$ & $5.92 \times 10^{13}$ \\
\hline \multirow[t]{2}{*}{2.6} & 1.7438 & 1.5733 & 1.2523 & 0.052 & 0.492 & 0.321 & 0.0227 & $3.31 \times 10^{13}$ & $2.65 \times 10^{13}$ \\
\hline & & 1.6222 & 1.3012 & 0.067 & 0.443 & & 0.0116 & $8.47 \times 10^{13}$ & $5.41 \times 10^{13}$ \\
\hline \multirow[t]{2}{*}{2.8} & 1.7465 & 1.5600 & 1.2350 & 0.050 & 0.512 & 0.325 & 0.0269 & $2.59 \times 10^{13}$ & $2.34 \times 10^{13}$ \\
\hline & & 1.6062 & 1.2812 & 0.065 & 0.465 & & 0.0151 & $6.93 \times 10^{13}$ & $4.61 \times 10^{13}$ \\
\hline \multirow[t]{2}{*}{3.0} & 1.7480 & 1.5462 & 1.2202 & 0.048 & 0.528 & 0.326 & 0.0312 & $2.01 \times 10^{13}$ & $2.07 \times 10^{13}$ \\
\hline & & 1.5911 & 1.2651 & 0.064 & 0.483 & & 0.0003 & $5.78 \times 10^{13}$ & $4.19 \times 10^{13}$ \\
\hline \multirow[t]{2}{*}{3.5} & 1.7527 & 1.5207 & 1.1937 & 0.044 & 0.559 & 0.327 & 0.0411 & $1.15 \times 10^{13}$ & $1.57 \times 10^{13}$ \\
\hline & & 1.5654 & 1.2384 & 0.059 & 0.514 & & 0.0015 & $3.62 \times 10^{13}$ & $3.28 \times 10^{13}$ \\
\hline \multirow[t]{2}{*}{4.0} & 1.7560 & 1.5022 & 1.1702 & 0.042 & 0.586 & 0.332 & 0.0486 & $1.09 \times 10^{13}$ & $1.45 \times 10^{13}$ \\
\hline & & 1.5477 & 1.2157 & 0.058 & 0.540 & & 0.0067 & $1.86 \times 10^{13}$ & $2.87 \times 10^{13}$ \\
\hline \multirow[t]{2}{*}{5.0} & 1.7600 & 1.4757 & 1.1437 & 0.039 & 0.616 & 0.332 & 0.0607 & $4.22 \times 10^{12}$ & $9.26 \times 10^{12}$ \\
\hline & & 1.5226 & 1.1906 & 0.050 & 0.569 & & 0.0423 & $1.42 \times 10^{13}$ & $1.90 \times 10^{13}$ \\
\hline \multirow[t]{2}{*}{10.0} & 1.7573 & 1.4115 & 1.0685 & 0.033 & 0.689 & 0.343 & 0.0872 & $1.07 \times 10^{12}$ & $5.12 \times 10^{12}$ \\
\hline & & 1.4409 & 1.0979 & 0.050 & 0.659 & & 0.0223 & $4.28 \times 10^{12}$ & $1.36 \times 10^{13}$ \\
\hline \multirow[t]{2}{*}{78.0} & 1.7534 & 1.3584 & 1.0094 & 0.027 & 0.744 & 0.349 & 0.1118 & $2.74 \times 10^{11}$ & $2.77 \times 10^{12}$ \\
\hline & & 1.3773 & 1.0283 & 0.048 & 0.725 & & 0.1013 & $1.30 \times 10^{12}$ & $9.63 \times 10^{12}$ \\
\hline
\end{tabular}




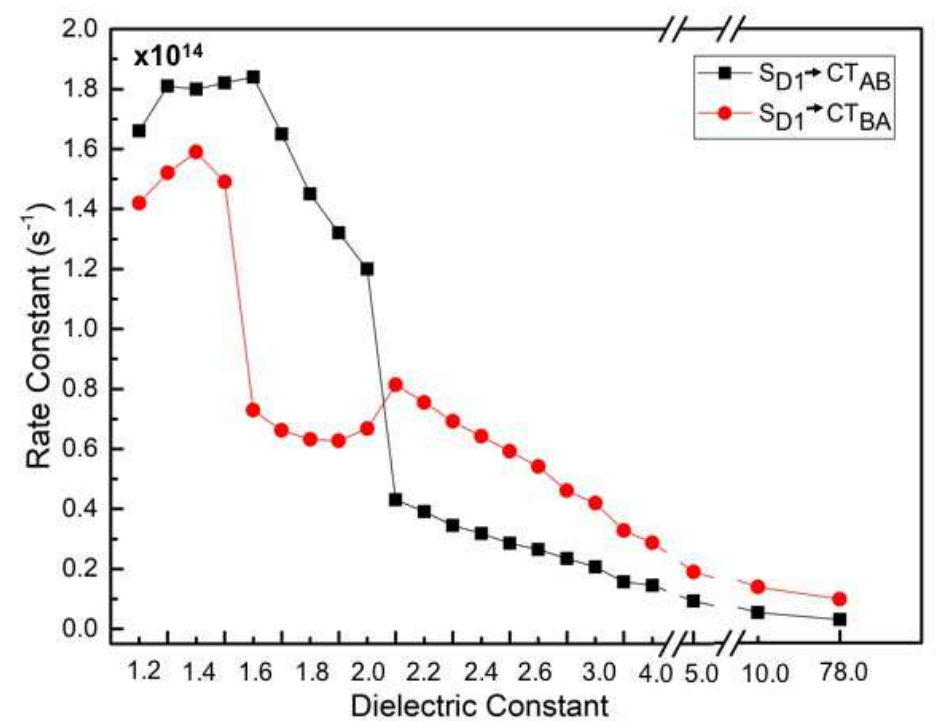

SI Figure S1. FGR CT rates for the full range of dielectric constants up to 78 . Black and red curves correspond to $\mathrm{CT}_{A B}$ and $\mathrm{CT}_{B A}$ processes, respectively.

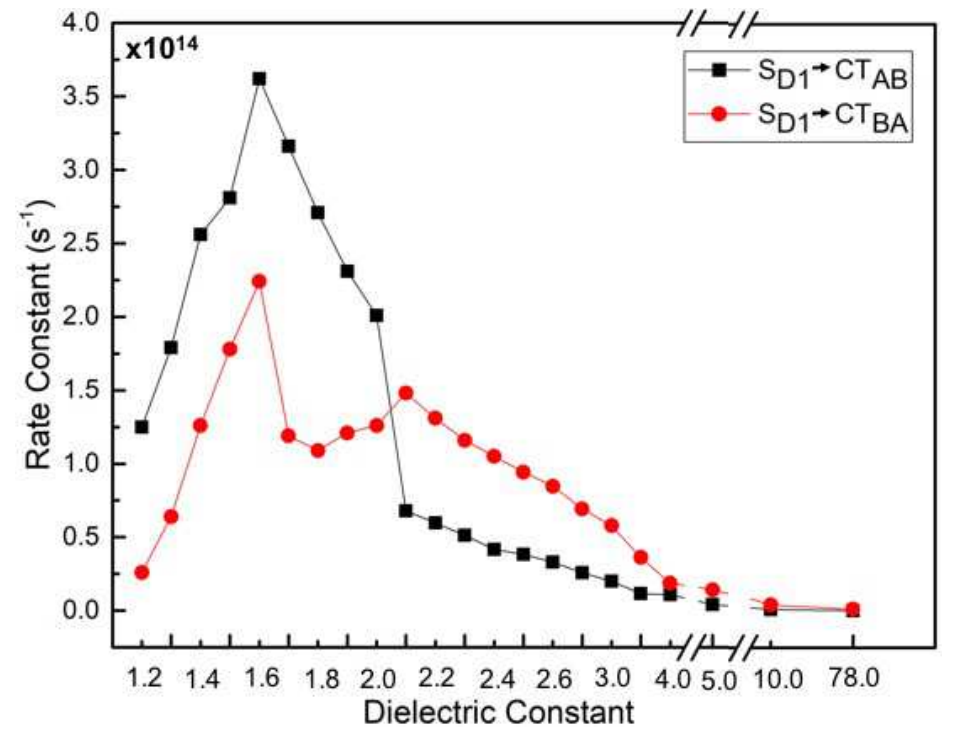

SI Figure S2. Marcus CT rates for the full range of dielectric constants up to 78. Black and red curves correspond to $\mathrm{CT}_{A B}$ and $\mathrm{CT}_{B A}$ processes, respectively 

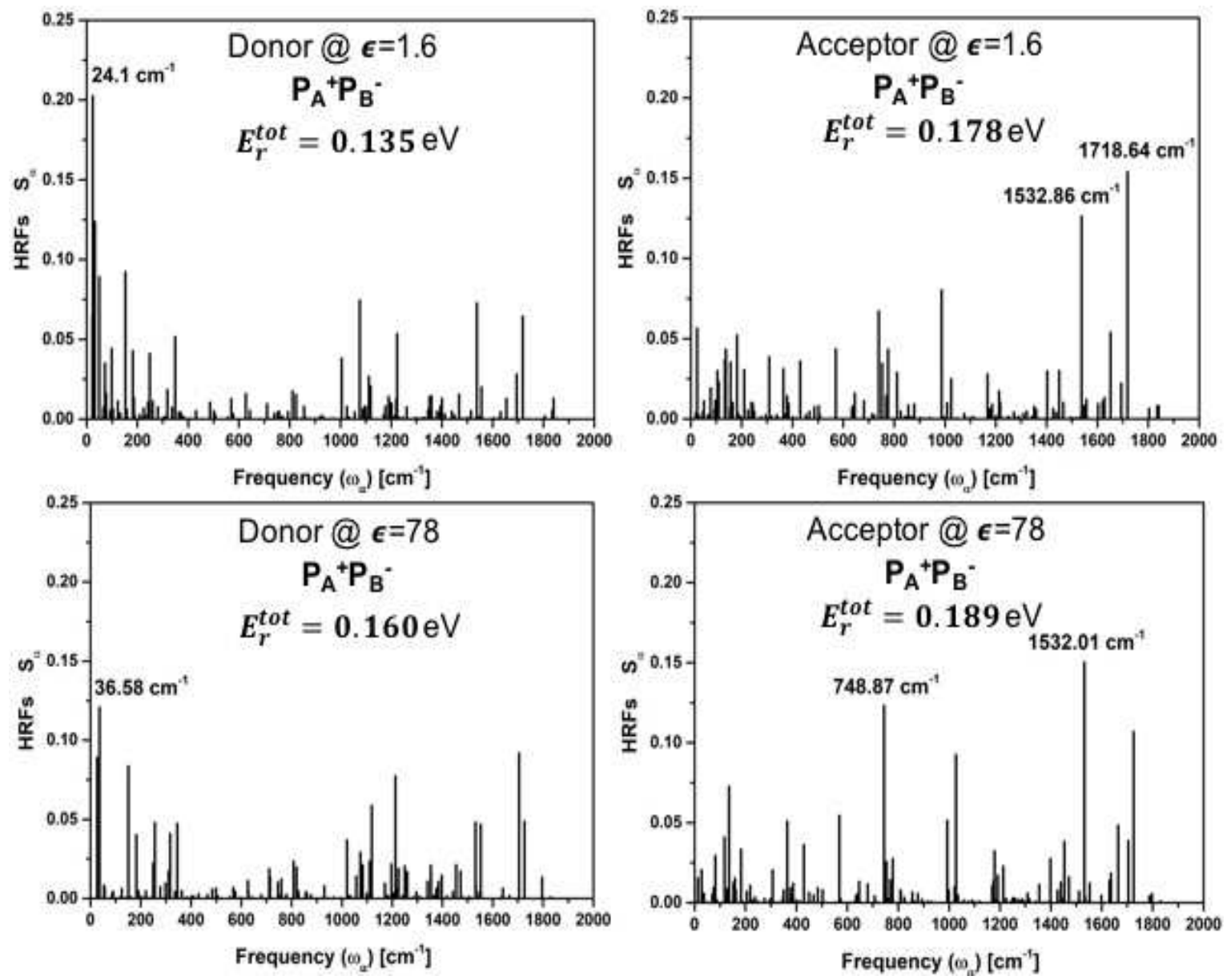

SI Figure S3. HRF distributions due to electron transfer $\left(\mathrm{P}_{A}^{+} \mathrm{P}_{B}^{-}\right)$in the special pair for dielectric constants 1.6 (upper panel) and 78 (lower panel) due to acceptor and donor monomer contributions. 

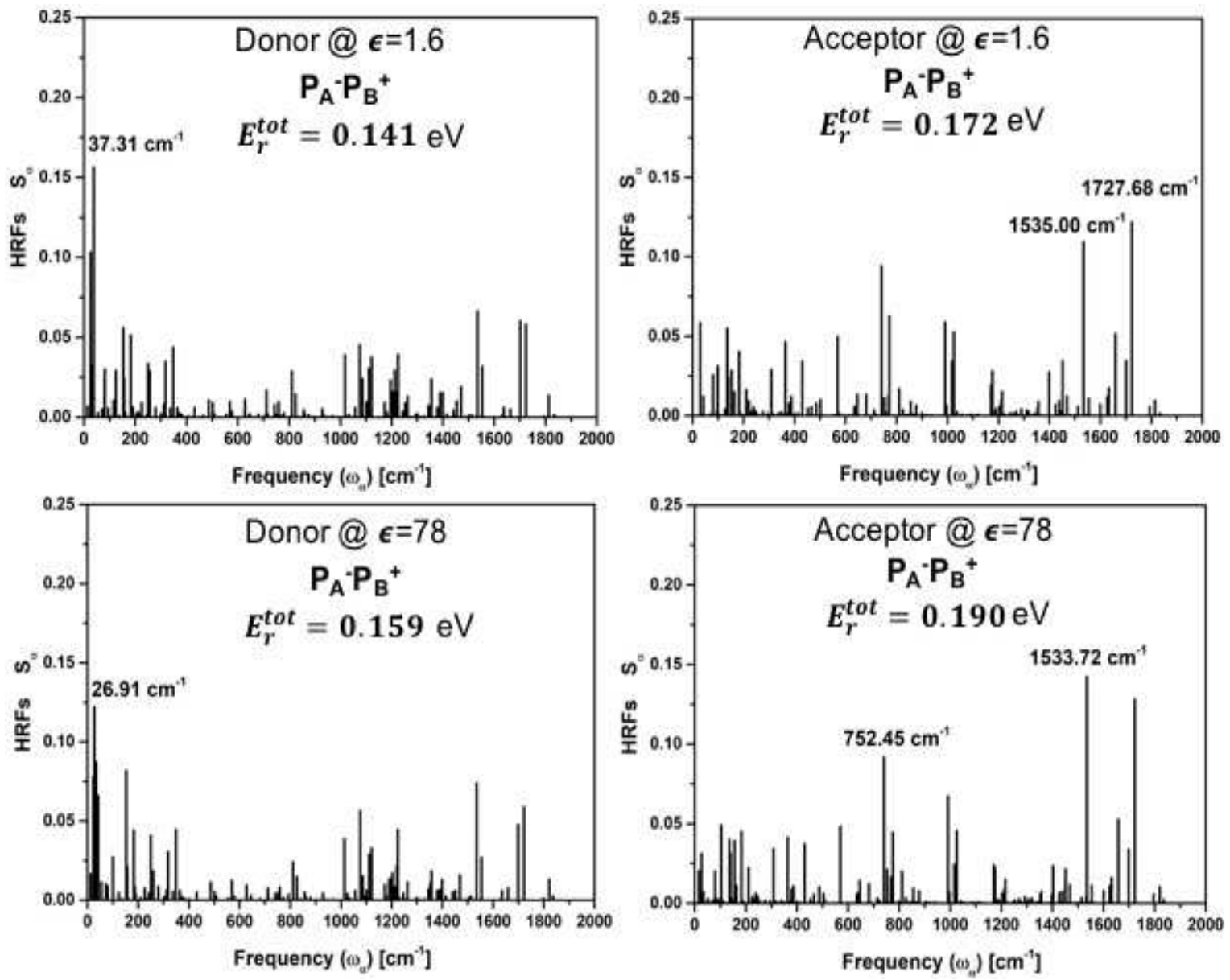

SI Figure S4. HRF distributions due to electron transfer $\left(\mathrm{P}_{A}^{-} \mathrm{P}_{B}^{+}\right)$in the special pair for dielectric constants 1.6 (upper panel) and 78 (lower panel) due to acceptor and donor monomer contributions. 


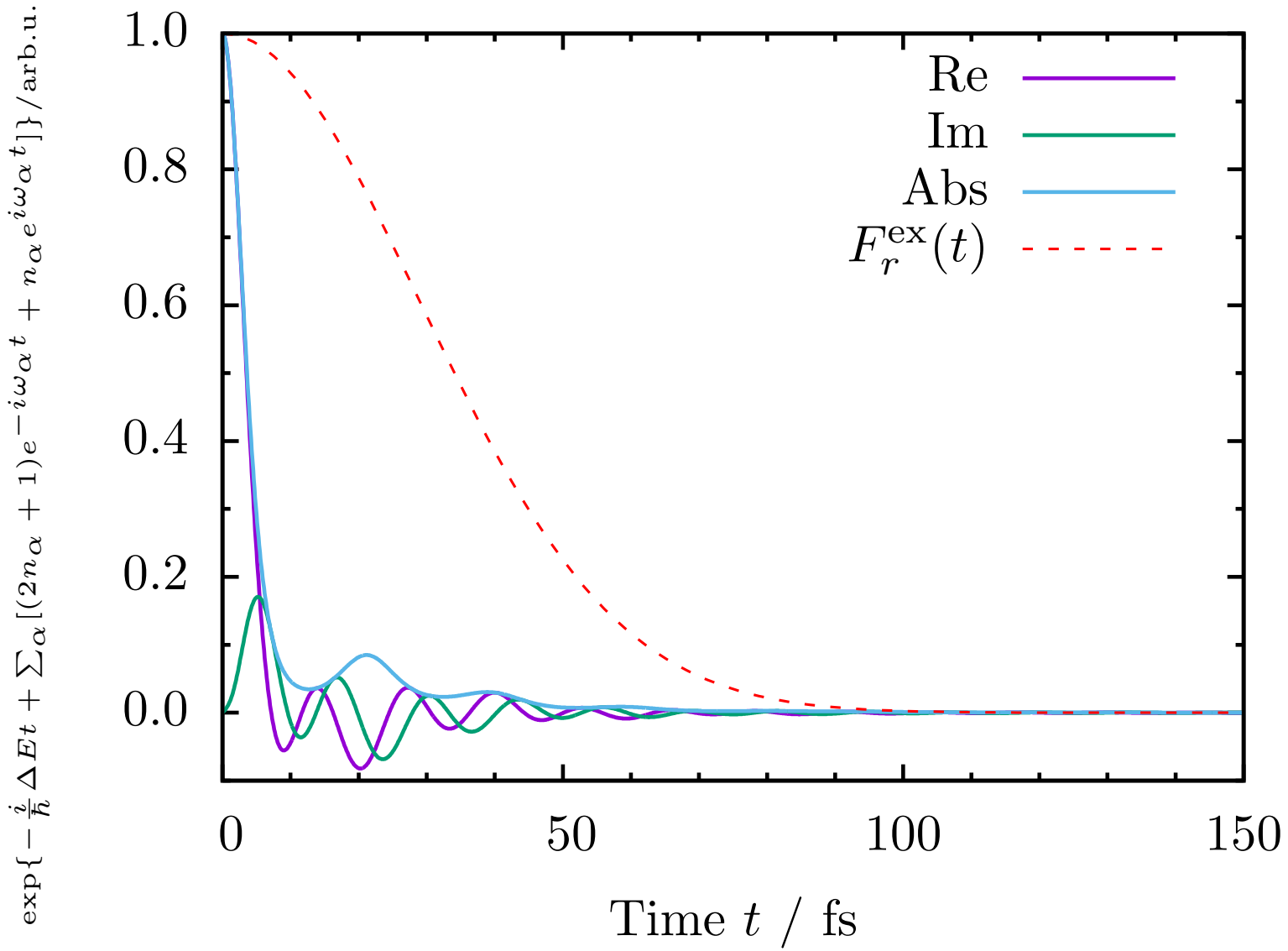

SI Figure S5. The time-dependent integrand of the FGR-expression (solid lines), without the $F_{r}^{\text {ex }}(t)$ window function, decays at $\epsilon=1.6$ (highest rate) on a sub-100 fs timescale. The semi-classical contribution of an external reorganization energy $E^{\mathrm{ex}}$ of $0.01 \mathrm{eV}$ leads to a window function $F_{r}^{\text {ex }}(t)$ (red dashed line) with only negligible effect on the FGR rate. 
SI Table S4. The atomic coordinates of the X-Ray structure of special pair $(\AA)$.

\begin{tabular}{|c|c|c|c|c|c|c|c|}
\hline $\mathrm{Mg}$ & -4.285600000000000 & 4.1406700000000001 & -3.9274900000000001 & $\mathrm{Mg}$ & -9.747600000000000 & -0.1673300000000000 & 0.0385100000000000 \\
\hline $\mathrm{C}$ & -0.978600000000000 & 3.5786699999999998 & -4.8334900000000003 & $\mathrm{C}$ & -12.562600000000000 & -2.0153300000000001 & -0.9554900000000000 \\
\hline $\mathrm{C}$ & -5.054600000000000 & 0.9996699999999999 & -5.1644899999999998 & $\mathrm{C}$ & -7.827600000000000 & -2.1383299999999998 & -2.0474899999999998 \\
\hline $\mathrm{C}$ & -7.349600000000000 & 4.2586700000000004 & -2.3654899999999999 & $\mathrm{C}$ & -7.185600000000000 & 2.1506699999999999 & 0.1625100000000000 \\
\hline $\mathrm{C}$ & -3.107600000000000 & 6.6666699999999999 & -1.7944899999999999 & $\mathrm{C}$ & -11.905600000000000 & 2.1856700000000000 & 1.4395100000000001 \\
\hline $\mathrm{N}$ & -3.183600000000000 & 2.5906699999999998 & -4.9374900000000004 & $\mathrm{~N}$ & -10.110600000000000 & -1.9173300000000000 & -1.1684900000000000 \\
\hline $\mathrm{C}$ & -1.851600000000000 & 2.6466699999999999 & -5.3034900000000000 & $\mathrm{C}$ & -11.355600000000001 & -2.5283300000000000 & -1.3974899999999999 \\
\hline $\mathrm{C}$ & -1.561600000000000 & 1.5626700000000000 & -6.3024899999999997 & $\mathrm{C}$ & -11.160600000000001 & -3.6923300000000001 & -2.3084899999999999 \\
\hline $\mathrm{C}$ & -2.767600000000000 & 0.6706700000000000 & -6.2544899999999997 & $\mathrm{C}$ & -9.669600000000001 & -3.9023300000000001 & -2.2834900000000000 \\
\hline $\mathrm{C}$ & -3.754600000000000 & 1.4086700000000001 & -5.3894900000000003 & $\mathrm{C}$ & -9.119600000000000 & -2.5673300000000001 & -1.8704900000000000 \\
\hline $\mathrm{C}$ & -0.325600000000000 & 0.7356700000000000 & -6.0104899999999999 & $\mathrm{C}$ & -11.621600000000001 & -3.3353299999999999 & -3.7214900000000002 \\
\hline $\mathrm{C}$ & -0.159600000000000 & -0.4323300000000000 & -6.9514899999999997 & $\mathrm{C}$ & -11.654600000000000 & -4.4413299999999998 & -4.7204899999999999 \\
\hline $\mathrm{C}$ & 0.831400000000000 & -1.4203300000000001 & -6.4334899999999999 & $\mathrm{C}$ & -12.848599999999999 & -5.3313300000000003 & -4.5364899999999997 \\
\hline $\mathrm{O}$ & 0.607400000000000 & -2.6133299999999999 & -6.5784900000000004 & $\mathrm{O}$ & -13.459600000000000 & -5.3023300000000004 & -3.4584899999999998 \\
\hline $\mathrm{O}$ & 1.969400000000000 & -1.0423300000000000 & -5.7294900000000002 & $\mathrm{O}$ & -13.298600000000000 & -6.2343299999999999 & -5.5794899999999998 \\
\hline $\mathrm{N}$ & -5.933600000000000 & 2.9086699999999999 & -3.8534899999999999 & $\mathrm{~N}$ & -7.833600000000000 & -0.0643300000000000 & -0.7264900000000000 \\
\hline $\mathrm{C}$ & -6.043600000000000 & 1.6746700000000001 & 54900000000000 & $\mathrm{C}$ & -7.240600000000000 & -0.9973300000000000 & -1.5244899999999999 \\
\hline $\mathrm{C}$ & -7.386600000000000 & 1.1766700000000001 & -4.2814899999999998 & $\mathrm{C}$ & -5.873600000000000 & -0.6153300000000000 & -1.7184900000000001 \\
\hline $\mathrm{C}$ & -8.054600000000001 & 2.0616699999999999 & -3.4804900000000001 & $\mathrm{C}$ & -5.743600000000000 & 0.7106700000000000 & -1.3704900000000000 \\
\hline $\mathrm{C}$ & -7.113600000000000 & 3.1486700000000001 & -3.1774900000000001 & $\mathrm{C}$ & -6.956600000000000 & 0.9866700000000000 & -0.5914900000000000 \\
\hline $\mathrm{C}$ & -9.365600000000001 & 1.9616700000000000 & -2.8414899999999998 & $\mathrm{C}$ & -4.713600000000000 & 1.5856699999999999 & -0.8254899999999999 \\
\hline $\mathrm{O}$ & -10.094600000000000 & 0.9626700000000000 & -2.8494899999999999 & $\mathrm{O}$ & -4.000600000000000 & 1.3196699999999999 & 0.0875100000000000 \\
\hline $\mathrm{C}$ & -10.018599999999999 & 3.2616700000000001 & -2.4964900000000001 & $\mathrm{C}$ & -3.914600000000000 & 1.8966700000000001 & -2.0634899999999998 \\
\hline $\mathrm{N}$ & -5.118600000000000 & 5.3536700000000002 & -2.4554900000000002 & $\mathrm{~N}$ & -9.534599999999999 & 1.7696700000000001 & 0.9025100000000000 \\
\hline $\mathrm{C}$ & -6.460600000000000 & 5.2806699999999998 & -2.0494900000000000 & $\mathrm{C}$ & -8.350600000000000 & 2.5286700000000000 & 0.8515100000000000 \\
\hline $\mathrm{C}$ & -6.789600000000000 & 6.5676699999999997 & -1.3304900000000000 & $\mathrm{C}$ & -8.526600000000000 & 3.7686700000000002 & 1.7095100000000001 \\
\hline $\mathrm{C}$ & -5.463600000000000 & 6.9576700000000002 & -0.7454900000000000 & $\mathrm{C}$ & -10.029600000000000 & 3.9256700000000002 & 1.7955099999999999 \\
\hline $\mathrm{C}$ & -4.467600000000000 & 6.3646700000000003 & -1.7254900000000000 & $\mathrm{C}$ & -10.565600000000000 & 2.5416699999999999 & 1.4665100000000000 \\
\hline $\mathrm{N}$ & -2.504600000000000 & 4.9356700000000000 & -3.3694899999999999 & $\mathrm{~N}$ & -11.725600000000000 & 0.0366700000000000 & 0.3275100000000000 \\
\hline $\mathrm{C}$ & -2.168600000000000 & 5.9976700000000003 & -2.5664899999999999 & $\mathrm{C}$ & -12.467599999999999 & 1.0276700000000001 & 0.9105100000000000 \\
\hline $\mathrm{C}$ & -0.739600000000000 & 6.2876700000000003 & -2.6424900000000000 & $\mathrm{C}$ & -13.889600000000000 & 0.6796700000000000 & 0.8805100000000000 \\
\hline $\mathrm{C}$ & -0.252600000000000 & 5.4276700000000000 & -3.5684900000000002 & $\mathrm{C}$ & -13.980600000000001 & -0.4313300000000000 & 0.1125100000000000 \\
\hline $\mathrm{C}$ & -1.324600000000000 & & -3.9414899999999999 & $\mathrm{C}$ & -12.645600000000000 & -0.8553300000000000 & -0.1824900000000000 \\
\hline $\mathrm{C}$ & 0.890400000000000 & 5.0406700000000004 & -4.3484900000000000 & $\mathrm{C}$ & -14.873600000000000 & -1.3423300000000000 & -0.5654900000000000 \\
\hline $\mathrm{O}$ & 2.064400000000000 & 5.4646699999999999 & -4.3504899999999997 & $\mathrm{O}$ & -16.102599999999999 & -1.4343300000000001 & -0.5334900000000000 \\
\hline $\mathrm{C}$ & 0.419400000000000 & 3.9516700000000000 & -5.3264899999999997 & $\mathrm{C}$ & -14.014600000000000 & -2.3323299999999998 & -1.3804900000000000 \\
\hline $\mathrm{C}$ & 0.239400000000000 & 4.5876700000000001 & -6.6664899999999996 & $\mathrm{C}$ & -14.390599999999999 & -3.7433299999999998 & -0.9714900000000000 \\
\hline $\mathrm{O}$ & 0.837400000000000 & 4.0656699999999999 & -7.6194899999999999 & $\mathrm{O}$ & -13.642600000000000 & -4.3553300000000004 & -0.1924900000000000 \\
\hline $\mathrm{O}$ & -0.576600000000000 & 5.6456700000000000 & -6.8344899999999997 & $\mathrm{O}$ & -15.585599999999999 & -4.2893299999999996 & -1.3904900000000000 \\
\hline $\mathrm{C}$ & -0.759600000000000 & 6.2616699999999996 & -8.0714900000000007 & $\mathrm{C}$ & -16.049600000000002 & -5.4153300000000000 & -0.8634900000000000 \\
\hline $\mathrm{C}$ & 2.627400000000000 & -2.0983299999999998 & -4.9834899999999998 & $\mathrm{C}$ & -14.679600000000001 & -6.6613300000000004 & -5.5124899999999997 \\
\hline $\mathrm{H}$ & -5.320610000000000 & 0.0856000000000000 & -5.5629000000000000 & $\mathrm{H}$ & -7.224940000000000 & -2.7333699999999999 & -2.6372000000000000 \\
\hline $\mathrm{H}$ & -8.292370000000000 & 4.3294800000000002 & -1.9517400000000000 & $\mathrm{H}$ & -6.396250000000000 & 2.8132299999999999 & 0.2171600000000000 \\
\hline $\mathrm{H}$ & -2.770530000000000 & & -1.2216800000000001 & $\mathrm{H}$ & -12.563879999999999 & 2.8591099999999998 & 1.8616200000000001 \\
\hline $\mathrm{H}$ & -1.367810000000000 & 2.0106299999999999 & -7.2546900000000001 & $\mathrm{H}$ & -11.713609999999999 & -4.5597899999999996 & -2.0142300000000000 \\
\hline $\mathrm{H}$ & -3.165030000000000 & 0.5202900000000000 & -7.2365000000000004 & $\mathrm{H}$ & -9.404170000000001 & -4.6584899999999996 & -1.5745000000000000 \\
\hline $\mathrm{H}$ & -2.537780000000000 & -0.3004400000000000 & -5.8684500000000002 & $\mathrm{H}$ & -9.284979999999999 & -4.2315800000000001 & -3.2261299999999999 \\
\hline $\mathrm{H}$ & 0.534370000000000 & 1.3666300000000000 & -6.0956799999999998 & $\mathrm{H}$ & -12.612019999999999 & -2.9372500000000001 & -3.6473300000000002 \\
\hline $\mathrm{H}$ & -0.433770000000000 & 0.3374600000000000 & -5.0232599999999996 & $\mathrm{H}$ & -10.887210000000000 & -2.6495199999999999 & -4.0892400000000002 \\
\hline $\mathrm{H}$ & -1.105020000000000 & -0.9190300000000000 & -7.0705900000000002 & $\mathrm{H}$ & -11.687410000000000 & -4.0183700000000000 & -5.7027999999999999 \\
\hline $\mathrm{H}$ & 0.199290000000000 & -0.0586700000000000 & -7.8876999999999997 & $\mathrm{H}$ & -10.775630000000000 & -5.0359400000000001 & -4.5835600000000003 \\
\hline $\mathrm{H}$ & -7.773340000000000 & 0.3080800000000000 & -4.6827300000000003 & $\mathrm{H}$ & -5.116800000000000 & -1.2281599999999999 & -2.0601200000000000 \\
\hline $\mathrm{H}$ & -9.290039999999999 & 4.0448800000000000 & -2.5230199999999998 & $\mathrm{H}$ & -4.580020000000000 & 2.1320299999999999 & -2.8676800000000000 \\
\hline $\mathrm{H}$ & -10.440049999999999 & 3.1997000000000000 & -1.5149400000000000 & $\mathrm{H}$ & -3.321760000000000 & 1.0462199999999999 & -2.3283700000000001 \\
\hline $\mathrm{H}$ & -10.793020000000000 & 3.4708000000000001 & -3.2046100000000002 & $\mathrm{H}$ & -3.274300000000000 & 2.7327599999999999 & -1.8740600000000001 \\
\hline $\mathrm{H}$ & -7.518820000000000 & 6.4074499999999999 & -0.5640300000000000 & $\mathrm{H}$ & -8.078469999999999 & 4.6222200000000004 & 1.2452399999999999 \\
\hline $\mathrm{H}$ & -7.213320000000000 & 7.3165800000000001 & -1.9664900000000001 & $\mathrm{H}$ & -8.060150000000000 & 3.6825199999999998 & 2.6686299999999998 \\
\hline $\mathrm{H}$ & -5.364580000000000 & 8.0218299999999996 & -0.6937400000000000 & $\mathrm{H}$ & -10.324830000000000 & 4.2233499999999999 & 2.7799499999999999 \\
\hline $\mathrm{H}$ & -5.326750000000000 & 6.6084100000000001 & 0.2566000000000000 & $\mathrm{H}$ & -10.402670000000001 & 4.6789500000000004 & 1.1334599999999999 \\
\hline $\mathrm{H}$ & -0.213910000000000 & 6.9954900000000002 & -2.1061399999999999 & $\mathrm{H}$ & -14.662660000000001 & 1.1783399999999999 & 1.3482000000000001 \\
\hline $\mathrm{H}$ & 1.096340000000000 & 3.1250200000000001 & -5.3838499999999998 & $\mathrm{H}$ & -14.147640000000001 & -2.2494700000000001 & -2.4389500000000002 \\
\hline $\mathrm{H}$ & -1.446890000000000 & 7.0750299999999999 & -7.9667000000000003 & $\mathrm{H}$ & -16.982730000000000 & -5.6662200000000000 & -1.3230700000000000 \\
\hline $\mathrm{H}$ & -1.151360000000000 & 5.5513100000000000 & -8.7691999999999997 & $\mathrm{H}$ & -16.194120000000002 & -5.2837399999999999 & 0.1885100000000000 \\
\hline $\mathrm{H}$ & 0.178990000000000 & 6.6315299999999997 & -8.4280899999999992 & $\mathrm{H}$ & -15.346090000000000 & -6.2039499999999999 & -1.0309699999999999 \\
\hline $\mathrm{H}$ & 3.485210000000000 & -1.7001900000000001 & -4.4829299999999996 & $\mathrm{H}$ & -14.889419999999999 & -7.3196099999999999 & -6.3295199999999996 \\
\hline $\mathrm{H}$ & 2.934010000000000 & -2.8722099999999999 & -5.6558000000000002 & $\mathrm{H}$ & -15.320449999999999 & -5.8063700000000003 & -5.5696500000000002 \\
\hline $\mathrm{H}$ & 1.948310000000000 & -2.5014400000000001 & -4.2615299999999996 & $\mathrm{H}$ & -14.850080000000000 & -7.1737299999999999 & -4.5887599999999997 \\
\hline
\end{tabular}


SI Table S5. The atomic coordinates of the X-Ray structure of $\mathrm{BChl}$ monomer $\left(P_{A}\right)$ molecule $(\AA)$.

\begin{tabular}{|c|c|c|c|}
\hline $\mathrm{Mg}$ & -9.747600000000000 & -0.1673300000000000 & 0.0385100000000000 \\
\hline $\mathrm{C}$ & -12.562600000000000 & -2.0153300000000001 & -0.9554900000000000 \\
\hline $\mathrm{C}$ & -7.827600000000000 & -2.1383299999999998 & -2.0474899999999998 \\
\hline $\mathrm{C}$ & -7.185600000000000 & 2.1506699999999999 & 0.1625100000000000 \\
\hline $\mathrm{C}$ & -11.905600000000000 & 2.1856700000000000 & 1.4395100000000001 \\
\hline $\mathrm{N}$ & -10.110600000000000 & -1.9173300000000000 & -1.168490000000000 \\
\hline $\mathrm{C}$ & -11.355600000000001 & -2.5283300000000000 & -1.3974899999999999 \\
\hline $\mathrm{C}$ & -11.160600000000001 & -3.6923300000000001 & -2.3084899999999999 \\
\hline $\mathrm{C}$ & -9.669600000000001 & -3.9023300000000001 & -2.2834900000000000 \\
\hline $\mathrm{C}$ & -9.119600000000000 & -2.5673300000000001 & -1.8704900000000000 \\
\hline $\mathrm{C}$ & -11.621600000000001 & -3.3353299999999999 & -3.7214900000000002 \\
\hline $\mathrm{C}$ & -11.654600000000000 & -4.4413299999999998 & -4.7204899999999999 \\
\hline $\mathrm{C}$ & -12.848599999999999 & -5.3313300000000003 & -4.5364899999999997 \\
\hline $\mathrm{O}$ & -13.459600000000000 & -5.3023300000000004 & -3.4584899999999998 \\
\hline $\mathrm{O}$ & -13.298600000000000 & -6.2343299999999999 & -5.5794899999999998 \\
\hline $\mathrm{N}$ & -7.833600000000000 & -0.0643300000000000 & -0.7264900000000000 \\
\hline $\mathrm{C}$ & -7.240600000000000 & -0.9973300000000000 & -1.5244899999999999 \\
\hline $\mathrm{C}$ & -5.873600000000000 & -0.6153300000000000 & -1.7184900000000001 \\
\hline $\mathrm{C}$ & -5.743600000000000 & 0.7106700000000000 & -1.3704900000000000 \\
\hline $\mathrm{C}$ & -6.956600000000000 & 0.9866700000000000 & -0.5914900000000000 \\
\hline $\mathrm{C}$ & -4.713600000000000 & 1.5856699999999999 & -0.8254899999999999 \\
\hline $\mathrm{O}$ & -4.000600000000000 & 1.3196699999999999 & 0.0875100000000000 \\
\hline $\mathrm{C}$ & -3.914600000000000 & 1.8966700000000001 & -2.0634899999999998 \\
\hline $\mathrm{N}$ & -9.534599999999999 & 1.7696700000000001 & 0.9025100000000000 \\
\hline $\mathrm{C}$ & -8.350600000000000 & 2.5286700000000000 & 0.8515100000000000 \\
\hline $\mathrm{C}$ & -8.526600000000000 & 3.7686700000000002 & 1.7095100000000001 \\
\hline $\mathrm{C}$ & -10.029600000000000 & 3.9256700000000002 & 1.7955099999999999 \\
\hline $\mathrm{C}$ & -10.565600000000000 & 2.5416699999999999 & 1.4665100000000000 \\
\hline N & -11.725600000000000 & 0.0366700000000000 & 0.3275100000000000 \\
\hline $\mathrm{C}$ & -12.467599999999999 & 1.0276700000000001 & 0.9105100000000000 \\
\hline $\mathrm{C}$ & -13.889600000000000 & & \\
\hline $\mathrm{C}$ & -13.980600000000001 & -0.4313300000000000 & 0.1125100000000000 \\
\hline $\mathrm{C}$ & -12.645600000000000 & -0.8553300000000000 & 900000000000 \\
\hline $\mathrm{C}$ & -14.873600000000000 & -1.3423300000000000 & 4900000000000 \\
\hline $\mathrm{O}$ & -16.102599999999999 & -1.4343300000000001 & -0.5334900000000000 \\
\hline $\mathrm{C}$ & -14.014600000000000 & -2.3323299999999998 & -1.3804900000000000 \\
\hline $\mathrm{C}$ & -14.390599999999999 & -3.7433299999999998 & -0.9714900000000000 \\
\hline $\mathrm{O}$ & -13.642600000000000 & -4.3553300000000004 & -0.1924900000000000 \\
\hline $\mathrm{O}$ & -15.585599999999999 & -4.2893299999999996 & -1.3904900000000000 \\
\hline $\mathrm{C}$ & -16.049600000000002 & -5.4153300000000000 & -0.8634900000000000 \\
\hline $\mathrm{C}$ & -14.679600000000001 & -6.6613300000000004 & -5.5124899999999997 \\
\hline $\mathrm{H}$ & -7.224940000000000 & -2.7333699999999999 & -2.6372000000000000 \\
\hline $\mathrm{H}$ & -6.396250000000000 & 2.8132299999999999 & 0.2171600000000000 \\
\hline $\mathrm{H}$ & -12.563879999999999 & 2.8591099999999998 & 1.8616200000000001 \\
\hline $\mathrm{H}$ & -11.713609999999999 & -4.5597899999999996 & -2.0142300000000000 \\
\hline $\mathrm{H}$ & -9.404170000000001 & -4.6584899999999996 & -1.5745000000000000 \\
\hline $\mathrm{H}$ & -9.284979999999999 & -4.2315800000000001 & -3.2261299999999999 \\
\hline $\mathrm{H}$ & -12.612019999999999 & -2.9372500000000001 & -3.6473300000000002 \\
\hline $\mathrm{H}$ & -10.887210000000000 & -2.6495199999999999 & -4.0892400000000002 \\
\hline $\mathrm{H}$ & -11.687410000000000 & -4.0183700000000000 & -5.7027999999999999 \\
\hline $\mathrm{H}$ & -10.775630000000000 & -5.0359400000000001 & -4.5835600000000003 \\
\hline $\mathrm{H}$ & -5.116800000000000 & -1.2281599999999999 & -2.0601200000000000 \\
\hline $\mathrm{H}$ & -4.580020000000000 & 2.1320299999999999 & -2.8676800000000000 \\
\hline $\mathrm{H}$ & -3.321760000000000 & 1.0462199999999999 & -2.3283700000000001 \\
\hline $\mathrm{H}$ & -3.274300000000000 & 2.7327599999999999 & -1.8740600000000001 \\
\hline $\mathrm{H}$ & -8.078469999999999 & 4.6222200000000004 & 1.2452399999999999 \\
\hline $\mathrm{H}$ & -8.060150000000000 & 3.6825199999999998 & 2.6686299999999998 \\
\hline $\mathrm{H}$ & -10.324830000000000 & 4.2233499999999999 & 2.7799499999999999 \\
\hline $\mathrm{H}$ & -10.402670000000001 & 4.6789500000000004 & 1.1334599999999999 \\
\hline $\mathrm{H}$ & -14.662660000000001 & 1.1783399999999999 & 1.3482000000000001 \\
\hline $\mathrm{H}$ & -14.147640000000001 & -2.2494700000000001 & -2.4389500000000002 \\
\hline $\mathrm{H}$ & -16.982730000000000 & -5.6662200000000000 & -1.3230700000000000 \\
\hline $\mathrm{H}$ & -16.194120000000002 & -5.2837399999999999 & 0.1885100000000000 \\
\hline $\mathrm{H}$ & -15.346090000000000 & -6.2039499999999999 & -1.0309699999999999 \\
\hline $\mathrm{H}$ & -14.889419999999999 & -7.3196099999999999 & -6.3295199999999996 \\
\hline $\mathrm{H}$ & -15.320449999999999 & -5.8063700000000003 & -5.5696500000000002 \\
\hline $\mathrm{H}$ & -14.850080000000000 & -7.1737299999999999 & -4.5887599999999997 \\
\hline
\end{tabular}

SI Table S6. The atomic coordinates of the X-Ray structure of $\mathrm{BChl}$ monomer $\left(P_{B}\right)$ molecule $(\AA)$.

\begin{tabular}{|c|c|c|c|}
\hline $\mathrm{Mg}$ & -4.285600000000000 & 4.1406700000000001 & -3.9274900000000001 \\
\hline $\mathrm{C}$ & -0.978600000000000 & 3.5786699999999998 & -4.8334900000000003 \\
\hline $\mathrm{C}$ & -5.054600000000000 & 0.9996699999999999 & -5.1644899999999998 \\
\hline $\mathrm{C}$ & -7.349600000000000 & 4.2586700000000004 & -2.3654899999999999 \\
\hline $\mathrm{C}$ & -3.107600000000000 & 6.6666699999999999 & -1.7944899999999999 \\
\hline $\mathrm{N}$ & -3.183600000000000 & 2.5906699999999998 & -4.9374900000000004 \\
\hline $\mathrm{C}$ & -1.851600000000000 & 2.6466699999999999 & -5.3034900000000000 \\
\hline $\mathrm{C}$ & -1.561600000000000 & 1.5626700000000000 & -6.3024899999999997 \\
\hline $\mathrm{C}$ & -2.767600000000000 & 0.6706700000000000 & -6.2544899999999997 \\
\hline $\mathrm{C}$ & -3.754600000000000 & 1.4086700000000001 & -5.3894900000000003 \\
\hline $\mathrm{C}$ & -0.325600000000000 & 0.7356700000000000 & -6.0104899999999999 \\
\hline $\mathrm{C}$ & -0.159600000000000 & -0.4323300000000000 & -6.9514899999999997 \\
\hline $\mathrm{C}$ & 0.831400000000000 & -1.4203300000000001 & -6.4334899999999999 \\
\hline $\mathrm{O}$ & 0.607400000000000 & -2.6133299999999999 & -6.5784900000000004 \\
\hline $\mathrm{O}$ & 1.969400000000000 & -1.0423300000000000 & -5.7294900000000002 \\
\hline $\mathrm{N}$ & -5.933600000000000 & 2.9086699999999999 & -3.8534899999999999 \\
\hline $\mathrm{C}$ & -6.043600000000000 & 1.6746700000000001 & -4.4654900000000000 \\
\hline $\mathrm{C}$ & -7.386600000000000 & 1.1766700000000001 & -4.2814899999999998 \\
\hline $\mathrm{C}$ & -8.054600000000001 & 2.0616699999999999 & -3.4804900000000001 \\
\hline $\mathrm{C}$ & -7.113600000000000 & 3.1486700000000001 & -3.1774900000000001 \\
\hline $\mathrm{C}$ & -9.365600000000001 & 1.9616700000000000 & -2.8414899999999998 \\
\hline $\mathrm{O}$ & -10.094600000000000 & 0.9626700000000000 & -2.8494899999999999 \\
\hline $\mathrm{C}$ & -10.018599999999999 & 3.2616700000000001 & -2.4964900000000001 \\
\hline $\mathrm{N}$ & -5.118600000000000 & 5.3536700000000002 & -2.4554900000000002 \\
\hline $\mathrm{C}$ & -6.460600000000000 & 5.2806699999999998 & -2.0494900000000000 \\
\hline $\mathrm{C}$ & -6.789600000000000 & 6.5676699999999997 & -1.3304900000000000 \\
\hline $\mathrm{C}$ & -5.463600000000000 & 6.9576700000000002 & -0.7454900000000000 \\
\hline $\mathrm{C}$ & -4.467600000000000 & 6.3646700000000003 & -1.7254900000000000 \\
\hline $\mathrm{N}$ & -2.504600000000000 & 4.9356700000000000 & -3.3694899999999999 \\
\hline $\mathrm{C}$ & -2.168600000000000 & 5.9976700000000003 & -2.5664899999999999 \\
\hline $\mathrm{C}$ & -0.739600000000000 & 6.2876700000000003 & -2.6424900000000000 \\
\hline $\mathrm{C}$ & -0.252600000000000 & 5.4276700000000000 & -3.5684900000000002 \\
\hline $\mathrm{C}$ & -1.324600000000000 & 4.5446700000000000 & -3.9414899999999999 \\
\hline $\mathrm{C}$ & 0.890400000000000 & 5.0406700000000004 & -4.3484900000000000 \\
\hline $\mathrm{O}$ & 2.064400000000000 & 5.4646699999999999 & -4.3504899999999997 \\
\hline $\mathrm{C}$ & 0.419400000000000 & 3.9516700000000000 & -5.3264899999999997 \\
\hline $\mathrm{C}$ & 0.239400000000000 & 4.5876700000000001 & -6.6664899999999996 \\
\hline $\mathrm{O}$ & 0.837400000000000 & 4.0656699999999999 & -7.6194899999999999 \\
\hline $\mathrm{O}$ & -0.576600000000000 & 5.6456700000000000 & -6.8344899999999997 \\
\hline $\mathrm{C}$ & -0.759600000000000 & 6.2616699999999996 & -8.0714900000000007 \\
\hline $\mathrm{C}$ & 2.627400000000000 & -2.0983299999999998 & -4.9834899999999998 \\
\hline $\mathrm{H}$ & -5.320610000000000 & 0.0856000000000000 & -5.5629000000000000 \\
\hline $\mathrm{H}$ & -8.292370000000000 & 4.3294800000000002 & -1.9517400000000000 \\
\hline $\mathrm{H}$ & -2.770530000000000 & 7.4561700000000002 & -1.2216800000000001 \\
\hline $\mathrm{H}$ & -1.367810000000000 & 2.0106299999999999 & -7.2546900000000001 \\
\hline $\mathrm{H}$ & -3.165030000000000 & 0.5202900000000000 & -7.2365000000000004 \\
\hline $\mathrm{H}$ & -2.537780000000000 & -0.3004400000000000 & -5.8684500000000002 \\
\hline $\mathrm{H}$ & 0.534370000000000 & 1.3666300000000000 & -6.0956799999999998 \\
\hline $\mathrm{H}$ & -0.433770000000000 & 0.3374600000000000 & -5.0232599999999996 \\
\hline $\mathrm{H}$ & -1.105020000000000 & -0.9190300000000000 & -7.0705900000000002 \\
\hline $\mathrm{H}$ & 0.199290000000000 & -0.0586700000000000 & -7.8876999999999997 \\
\hline $\mathrm{H}$ & -7.773340000000000 & 0.3080800000000000 & -4.6827300000000003 \\
\hline $\mathrm{H}$ & -9.290039999999999 & 4.0448800000000000 & -2.5230199999999998 \\
\hline $\mathrm{H}$ & -10.440049999999999 & 3.1997000000000000 & -1.5149400000000000 \\
\hline $\mathrm{H}$ & -10.793020000000000 & 3.4708000000000001 & -3.2046100000000002 \\
\hline $\mathrm{H}$ & -7.518820000000000 & 6.4074499999999999 & -0.5640300000000000 \\
\hline $\mathrm{H}$ & -7.213320000000000 & 7.3165800000000001 & -1.9664900000000001 \\
\hline $\mathrm{H}$ & -5.364580000000000 & 8.0218299999999996 & -0.6937400000000000 \\
\hline $\mathrm{H}$ & -5.326750000000000 & 6.6084100000000001 & 0.2566000000000000 \\
\hline $\mathrm{H}$ & -0.213910000000000 & 6.9954900000000002 & -2.1061399999999999 \\
\hline $\mathrm{H}$ & 1.096340000000000 & 3.1250200000000001 & -5.3838499999999998 \\
\hline $\mathrm{H}$ & -1.446890000000000 & 7.0750299999999999 & -7.9667000000000003 \\
\hline $\mathrm{H}$ & -1.151360000000000 & 5.5513100000000000 & -8.7691999999999997 \\
\hline $\mathrm{H}$ & 0.178990000000000 & 6.6315299999999997 & -8.4280899999999992 \\
\hline $\mathrm{H}$ & 3.485210000000000 & -1.7001900000000001 & -4.4829299999999996 \\
\hline $\mathrm{H}$ & 2.934010000000000 & -2.8722099999999999 & -5.6558000000000002 \\
\hline H & 1.948310000000000 & -2.5014400000000001 & -4.2615299999999996 \\
\hline
\end{tabular}


SI Table S7. The atomic coordinates of structure demonstrated in Figure 5(b) $(\AA)$.

\begin{tabular}{|c|c|c|c|c|c|c|c|}
\hline $\mathrm{Mg}$ & 5.065200000000000 & -6.520420000000000 & -8.902910000000000 & $\mathrm{Mg}$ & 0.983660000000000 & -1.193780000000000 & -6.986180000000000 \\
\hline $\mathrm{C}$ & 5.207590000000000 & -9.885529999999999 & -8.058790000000000 & $\mathrm{C}$ & -2.405500000000000 & -0.988070000000000 & -6.407670000000000 \\
\hline $\mathrm{C}$ & 1.678220000000000 & -6.634730000000000 & -8.341070000000000 & $\mathrm{C}$ & 0.906500000000000 & -4.374470000000000 & -5.661050000000000 \\
\hline $\mathrm{C}$ & 4.727610000000000 & -3.437980000000000 & -10.323510000000001 & C & 4.372940000000000 & -1.329980000000000 & -7.141720000000000 \\
\hline $\mathrm{C}$ & 8.259480000000000 & -6.752640000000000 & -10.315910000000001 & $\mathrm{C}$ & 1.147400000000000 & 2.280720000000000 & -7.336490000000000 \\
\hline $\mathrm{N}$ & 3.655650000000000 & -8.057570000000000 & -8.228149999999999 & $\mathrm{~N}$ & -0.564970000000000 & -2.514400000000000 & -6.194710000000000 \\
\hline $\mathrm{C}$ & 3.969790000000000 & -9.364260000000000 & -7.851000000000000 & $\mathrm{C}$ & -1.931370000000000 & -2.235950000000000 & -6.168350000000000 \\
\hline $\mathrm{C}$ & 2.736710000000000 & -10.090890000000000 & -7.342350000000000 & $\mathrm{C}$ & -2.728040000000000 & -3.487950000000000 & -5.851660000000000 \\
\hline $\mathrm{C}$ & 1.652870000000000 & -9.002380000000000 & -7.409060000000000 & $\mathrm{C}$ & -1.629420000000000 & -4.428180000000000 & -5.320050000000000 \\
\hline $\mathrm{C}$ & 2.363270000000000 & -7.830260000000000 & -8.040310000000000 & $\mathrm{C}$ & -0.351080000000000 & -3.741410000000000 & -5.744560000000000 \\
\hline $\mathrm{N}$ & 3.464380000000000 & -5.311080000000000 & -9.395210000000001 & $\mathrm{~N}$ & 2.407700000000000 & -2.551080000000000 & -6.359230000000000 \\
\hline $\mathrm{C}$ & 2.194580000000000 & -5.496010000000000 & -8.928319999999999 & $\mathrm{C}$ & 2.136500000000000 & -3.838220000000000 & -5.992580000000000 \\
\hline $\mathrm{C}$ & 1.448270000000000 & -4.260220000000000 & -9.062720000000001 & $\mathrm{C}$ & 3.345360000000000 & -4.632380000000000 & -6.105680000000000 \\
\hline $\mathrm{C}$ & 2.287410000000000 & -3.339120000000000 & -9.600790000000000 & C & 4.326810000000000 & -3.812380000000000 & -6.558640000000000 \\
\hline $\mathrm{C}$ & 3.575430000000000 & -4.019360000000000 & -9.827240000000000 & $\mathrm{C}$ & 3.730180000000000 & -2.470730000000000 & -6.697730000000000 \\
\hline $\mathrm{N}$ & 6.303330000000000 & -5.289270000000000 & -10.180040000000000 & $\mathrm{~N}$ & 2.547090000000000 & 0.281440000000000 & -7.147970000000000 \\
\hline $\mathrm{C}$ & 5.985720000000000 & -4.053410000000000 & -10.522259999999999 & $\mathrm{C}$ & 3.822640000000000 & -0.031890000000000 & -7.283470000000000 \\
\hline $\mathrm{C}$ & 7.137660000000000 & -3.311510000000000 & -11.170500000000001 & $\mathrm{C}$ & 4.686140000000000 & 1.165810000000000 & -7.625600000000000 \\
\hline $\mathrm{C}$ & 8.253460000000000 & -4.366310000000000 & -11.206000000000000 & $\mathrm{C}$ & 3.675690000000000 & 2.324450000000000 & -7.655770000000000 \\
\hline $\mathrm{C}$ & 7.619770000000000 & -5.571890000000000 & -10.530510000000000 & $\mathrm{C}$ & 2.348550000000000 & 1.643200000000000 & -7.363540000000000 \\
\hline $\mathrm{N}$ & 6.456600000000000 & -7.973750000000000 & -9.169359999999999 & $\mathrm{~N}$ & -0.301390000000000 & 0.365050000000000 & -6.816280000000000 \\
\hline $\mathrm{C}$ & 7.735760000000000 & -7.935260000000000 & -9.693310000000000 & C & -0.148710000000000 & 1.711350000000000 & -7.090360000000000 \\
\hline $\mathrm{C}$ & 8.380929999999999 & -9.174189999999999 & -9.527490000000000 & C & -1.397690000000000 & 2.360350000000000 & -7.106520000000000 \\
\hline $\mathrm{C}$ & 7.443670000000000 & -9.996990000000000 & -8.873880000000000 & $\mathrm{C}$ & -2.347500000000000 & 1.354050000000000 & -6.840090000000000 \\
\hline $\mathrm{C}$ & 6.298930000000000 & -9.209840000000000 & -8.685510000000001 & C & -1.615040000000000 & 0.170410000000000 & -6.675300000000000 \\
\hline $\mathrm{C}$ & 7.200330000000000 & -11.313420000000001 & -8.334050000000000 & $\mathrm{C}$ & -3.752460000000000 & 1.049100000000000 & -6.682010000000000 \\
\hline $\mathrm{O}$ & 7.916750000000000 & -12.297689999999999 & -8.280989999999999 & $\mathrm{O}$ & -4.739480000000000 & 1.765510000000000 & -6.728420000000000 \\
\hline $\mathrm{C}$ & 5.724690000000000 & -11.283550000000000 & -7.750960000000000 & $\mathrm{C}$ & -3.849050000000000 & -0.500690000000000 & -6.418270000000000 \\
\hline $\mathrm{H}$ & 0.635330000000000 & -6.595750000000000 & -8.041639999999999 & $\mathrm{H}$ & 0.901960000000000 & -5.413540000000000 & -5.347240000000000 \\
\hline $\mathrm{H}$ & 4.681170000000000 & -2.397710000000000 & -10.620260000000000 & $\mathrm{H}$ & 5.426050000000000 & -1.406050000000000 & -7.384520000000000 \\
\hline $\mathrm{H}$ & 9.286470000000000 & -6.813630000000000 & -10.668410000000000 & $\mathrm{H}$ & 1.163410000000000 & 3.349740000000000 & -7.536570000000000 \\
\hline $\mathrm{H}$ & 2.509160000000000 & -10.878629999999999 & -8.068220000000000 & $\mathrm{H}$ & -3.484450000000000 & -3.288930000000000 & -5.085880000000000 \\
\hline $\mathrm{H}$ & 1.290630000000000 & -8.710640000000000 & -6.414520000000000 & $\mathrm{H}$ & -1.683100000000000 & -5.444560000000000 & -5.721860000000000 \\
\hline $\mathrm{H}$ & 7.400190000000000 & -2.431770000000000 & -10.572469999999999 & $\mathrm{H}$ & 5.186070000000000 & 1.010620000000000 & -8.587750000000000 \\
\hline $\mathrm{H}$ & 8.558270000000000 & -4.610160000000000 & -12.228540000000001 & $\mathrm{H}$ & 3.901330000000000 & 3.084550000000000 & -6.902020000000000 \\
\hline $\mathrm{H}$ & 5.820460000000000 & -11.468700000000000 & -6.675270000000000 & $\mathrm{H}$ & -4.435730000000000 & -0.950740000000000 & -7.225010000000000 \\
\hline $\mathrm{H}$ & 0.776730000000000 & -9.300739999999999 & -7.992220000000000 & $\mathrm{H}$ & 3.420640000000000 & -5.690480000000000 & -5.887360000000000 \\
\hline $\mathrm{H}$ & 0.418000000000000 & -4.118930000000000 & -8.760220000000000 & $\mathrm{H}$ & -1.559170000000000 & 3.415730000000000 & -7.286120000000000 \\
\hline $\mathrm{H}$ & 6.848550000000000 & -2.950410000000000 & -12.162580000000000 & $\mathrm{H}$ & -1.650730000000000 & -4.508370000000000 & -4.227130000000000 \\
\hline $\mathrm{H}$ & 9.150570000000000 & -4.040340000000000 & -10.671370000000000 & $\mathrm{H}$ & 5.468910000000000 & 1.299430000000000 & -6.872470000000000 \\
\hline $\mathrm{H}$ & 9.381539999999999 & -9.419790000000001 & -9.859299999999999 & $\mathrm{H}$ & 3.652470000000000 & 2.823080000000000 & -8.628869999999999 \\
\hline $\mathrm{H}$ & 2.830980000000000 & -10.527100000000001 & -6.369860000000000 & $\mathrm{H}$ & -3.272640000000000 & -3.882780000000000 & -6.683780000000000 \\
\hline $\mathrm{H}$ & 5.052390000000000 & -12.008620000000001 & -8.159829999999999 & $\mathrm{H}$ & -4.332740000000000 & -0.759440000000000 & -5.499580000000000 \\
\hline $\mathrm{H}$ & 2.066470000000000 & -2.353160000000000 & -9.810790000000001 & $\mathrm{H}$ & 5.302680000000000 & -4.076510000000000 & -6.765900000000000 \\
\hline
\end{tabular}


SI Table S8. The atomic coordinates of structure demonstrated in Figure 5(c) ( $\AA$ ).

\begin{tabular}{|c|c|c|c|c|c|c|c|}
\hline $\mathrm{Mg}$ & 4.681670112386202 & -6.263147802153376 & -8.642919665162513 & $\mathrm{Mg}$ & 6.602557400992416 & -6.887881603952142 & -6.446523257236870 \\
\hline $\mathrm{C}$ & 4.730720566929421 & -9.684315730269967 & -7.837267430732884 & $\mathrm{C}$ & 3.297973847750372 & -6.459162631615193 & -5.292917824277263 \\
\hline $\mathrm{C}$ & 1.251702788122455 & -6.434709870454210 & -8.486620030547767 & $\mathrm{C}$ & 6.683373192465033 & -9.802441350011572 & -4.635419174807075 \\
\hline $\mathrm{C}$ & 4.428080055271212 & -3.387776310741616 & -10.460169815820498 & $\mathrm{C}$ & 10.013198088796907 & -6.844357371644583 & -6.488864222793953 \\
\hline $\mathrm{C}$ & 8.006358115153320 & -6.581795012681004 & -9.826468070487380 & $\mathrm{C}$ & 6.653732661643051 & -3.463247751945231 & -7.312256882933360 \\
\hline $\mathrm{N}$ & 3.221421571753294 & -7.837626330432863 & -8.139795415629605 & $\mathrm{~N}$ & 5.138697700825863 & -7.998603847064761 & -5.212485159186647 \\
\hline $\mathrm{C}$ & 3.487744295029743 & -9.162113216216895 & -7.788211724839771 & $\mathrm{C}$ & 3.817886760002219 & -7.648536625589023 & -4.923767116659348 \\
\hline $\mathrm{C}$ & 2.210705781752119 & -9.904597811779963 & -7.466517057531822 & $\mathrm{C}$ & 3.134891819617389 & -8.728701585662893 & -4.114670062188452 \\
\hline $\mathrm{C}$ & 1.152365913162021 & -8.794785596314640 & -7.519049935897088 & $\mathrm{C}$ & 4.200706599306234 & -9.831415673135851 & -4.052948164429667 \\
\hline $\mathrm{C}$ & 1.917549684413736 & -7.615615074657518 & -8.089181944373992 & $\mathrm{C}$ & 5.418008967989281 & -9.172515758903932 & -4.670794737706442 \\
\hline $\mathrm{N}$ & 3.096724945793976 & -5.227426042980416 & -9.548443850374188 & $\mathrm{~N}$ & 8.097628302334325 & -8.005451750890444 & -5.501480527141549 \\
\hline $\mathrm{C}$ & 1.798945554848192 & -5.350623361010955 & -9.142275744842891 & $\mathrm{C}$ & 7.896625395856415 & -9.263188720270023 & -5.008995912837230 \\
\hline $\mathrm{C}$ & 1.062687787153023 & -4.131055970351833 & -9.480294685425386 & $\mathrm{C}$ & 9.176100794363839 & -9.974910866963178 & -4.957047263241190 \\
\hline $\mathrm{C}$ & 1.946496822083173 & -3.284415334698339 & -10.041026690351835 & C & 10.106539660699218 & -9.143869290219847 & -5.463998472083304 \\
\hline $\mathrm{C}$ & 3.243817759497623 & -3.964686618105522 & -10.050338233804862 & $\mathrm{C}$ & 9.415929752157167 & -7.909226265327106 & -5.843849735533272 \\
\hline $\mathrm{N}$ & 6.033170760741695 & -5.143445329793540 & -9.913311685445334 & $\mathrm{~N}$ & 8.096310667469661 & -5.386658003936782 & -6.879397773137692 \\
\hline $\mathrm{C}$ & 5.716714413113182 & -3.967182304280490 & -10.430570585823396 & $\mathrm{C}$ & 9.390493693452374 & -5.658005279823011 & -6.936927234858023 \\
\hline $\mathrm{C}$ & 6.920765454393149 & -3.241189688010459 & -10.993877540740447 & $\mathrm{C}$ & 10.184287703258166 & -4.541320437957602 & -7.584750424489431 \\
\hline $\mathrm{C}$ & 8.002376785177292 & -4.326392597322386 & -10.993494841065337 & $\mathrm{C}$ & 9.169369846259858 & -3.395160963168493 & -7.647737691271595 \\
\hline $\mathrm{C}$ & 7.371001579457968 & -5.437856829743915 & -10.173244782024653 & $\mathrm{C}$ & 7.859534757959295 & -4.078393904255023 & -7.298389716381434 \\
\hline $\mathrm{N}$ & 6.132175253227115 & -7.711845052522822 & -8.665099808409238 & $\mathrm{~N}$ & 5.275852696144304 & -5.329701291580429 & -6.452913100579930 \\
\hline $\mathrm{C}$ & 7.439450915177679 & -7.725545262548480 & -9.171190307945331 & C & 5.397358940549040 & -3.997821438273901 & -6.871066468402473 \\
\hline $\mathrm{C}$ & 8.009327378966429 & -8.995402326739764 & -9.037706479676253 & $\mathrm{C}$ & 4.191892009963001 & -3.310157613670424 & -6.693390549858547 \\
\hline $\mathrm{C}$ & 7.008955913897452 & -9.811924238374949 & -8.471835070400260 & $\mathrm{C}$ & 3.302150224254378 & -4.233070558290241 & -6.107202803318210 \\
\hline $\mathrm{C}$ & 5.897331226537328 & -8.996290419665449 & -8.286877906566597 & $\mathrm{C}$ & 4.010638593585860 & -5.422336123581426 & -5.966785394798322 \\
\hline $\mathrm{C}$ & 6.662184774836515 & -11.163634287212243 & -8.040336224799427 & $\mathrm{C}$ & 1.957361926543933 & -4.434195681420544 & -5.573018493120300 \\
\hline $\mathrm{O}$ & 7.349238645793972 & -12.167751410911430 & -7.992112398654382 & $\mathrm{O}$ & 1.008781312708722 & -3.671496983636784 & -5.540294027613346 \\
\hline $\mathrm{C}$ & 5.168489858528879 & -11.117345302949561 & -7.610279306371367 & $\mathrm{C}$ & 1.921766935486729 & -5.887158713053486 & -5.017615242884024 \\
\hline $\mathrm{H}$ & 0.188241783884702 & -6.390725212441435 & -8.271494080497009 & $\mathrm{H}$ & 6.701347244244525 & -10.810224585949445 & -4.231877861598499 \\
\hline $\mathrm{H}$ & 4.370865678414465 & -2.379535512020332 & -10.859134152636011 & $\mathrm{H}$ & 11.082374267518993 & -6.917899354892501 & -6.665284105312816 \\
\hline $\mathrm{H}$ & 9.045026630017778 & -6.676946883844566 & -10.133417248193405 & $\mathrm{H}$ & 6.633245071798373 & -2.430601909393331 & -7.651847407410062 \\
\hline $\mathrm{H}$ & 2.019509099711206 & -10.680848725245415 & -8.215948257610922 & $\mathrm{H}$ & 2.873726873102780 & -8.355512240347053 & -3.117934255954284 \\
\hline $\mathrm{H}$ & 0.772332206885941 & -8.529711972313292 & -6.524694656808907 & $\mathrm{H}$ & 3.924651686731669 & -10.710939679010618 & -4.647509963596705 \\
\hline $\mathrm{H}$ & 7.170589952964700 & -2.409541432876201 & -10.322679328334781 & $\mathrm{H}$ & 10.504145144412609 & -4.873384139043625 & -8.580699854849998 \\
\hline $\mathrm{H}$ & 8.207112332618482 & -4.682944807048016 & -12.009367728563934 & $\mathrm{H}$ & 9.399793309056838 & -2.621875088282586 & -6.906942557501343 \\
\hline $\mathrm{H}$ & 5.094843685161185 & -11.442762293723376 & -6.566258423598884 & $\mathrm{H}$ & 1.115923977308537 & -6.434713276838148 & -5.520389182727316 \\
\hline $\mathrm{H}$ & 0.286097711282160 & -9.045525440411762 & -8.137528800647029 & $\mathrm{H}$ & 9.299103440365045 & -10.983883878848520 & -4.585560268003750 \\
\hline $\mathrm{H}$ & 0.009376344650441 & -3.975047230976777 & -9.285536727689802 & $\mathrm{H}$ & 4.020374069818506 & -2.271463974131486 & -6.942612962985045 \\
\hline $\mathrm{H}$ & 6.720296968408930 & -2.816767859044978 & -11.981264036725435 & $\mathrm{H}$ & 4.408008607858338 & -10.183226743305575 & -3.038312696026619 \\
\hline $\mathrm{H}$ & 8.952903298656379 & -3.988489997637410 & -10.572503759483466 & $\mathrm{H}$ & 11.087822891459020 & -4.300028684239243 & -7.017884347235350 \\
\hline $\mathrm{H}$ & 9.004099056407393 & -9.276690369522784 & -9.356779863665558 & $\mathrm{H}$ & 9.132207402787055 & -2.910039945331830 & -8.626285876424587 \\
\hline $\mathrm{H}$ & 2.260163784517465 & -10.400701887585464 & -6.492917867256669 & $\mathrm{H}$ & 2.202845100081345 & -9.061198700262814 & -4.581197197652243 \\
\hline $\mathrm{H}$ & 4.594362468306144 & -11.832026860594315 & -8.212049097382748 & $\mathrm{H}$ & 1.672636390897452 & -5.861540342898767 & -3.949736381909533 \\
\hline $\mathrm{H}$ & 1.777486084130031 & -2.281118550798253 & -10.410820084441989 & $\mathrm{H}$ & 11.166932470908383 & -9.320999202371636 & -5.593296299762109 \\
\hline
\end{tabular}

